Electronic Journal of Statistics

Vol. 15 (2021) 2393-2429

ISSN: 1935-7524

https://doi.org/10.1214/21-EJS1836

\title{
A new family of copula-based concordance orderings of random pairs: Properties and nonparametric tests*
}

\author{
Jean-François Quessy \\ Université du Québec à Trois-Rivières \\ e-mail: Jean-Francois.Quessy@uqtr.ca \\ Mhamed Mesfioui \\ Université du Québec à Trois-Rivières \\ e-mail: Mhamed.Mesfioui@uqtr.ca
}

\begin{abstract}
The formal assessment of the stochastic dominance of a random pair with respect to another one is a question of interest in the economic analysis of populations. For example, a manager may wonder if the components of a portfolio are more associated than that of another competing portfolio, in which case the former is generally considered more at risk. In this paper, a new family of copula-based concordance orderings in the spirit of increasing convex and concave orderings of random pairs is introduced as a natural extension of the well-known concordance ordering. In addition, a complete statistical methodology to test the stochastic dominance of a random pair with respect to another one according to the new concordance orderings is developed. The proposed tests are nonparametric, consistent against all alternatives, and valid under serially dependent data satisfying the $\alpha$-mixing assumption. The sampling properties of the tests are investigated with the help of Monte-Carlo simulations and their usefulness is illustrated on real multivariate data.
\end{abstract}

MSC2020 subject classifications: Primary 60E15; secondary 62G10. Keywords and phrases: Bivariate time series, tests of composite hypotheses, s-increasing stochastic orders, concordance order, empirical copula, serial multiplier.

Received July 2020.

\section{Introduction}

Stochastic dominance is a key concept in the economic analysis of populations that allows to compare, for instance, incomes, poverty rankings and earnings. The most basic notion is that of first order stochastic dominance of a random variable $Y$ over another variable $X$, which is defined as $\mathrm{E}\{U(Y)\} \geq \mathrm{E}\{U(X)\}$ for all utility functions $U$ that are monotonically increasing; it can be shown to be equivalent to $\mathbb{P}(Y \leq x) \leq \mathbb{P}(X \leq x)$ for all $x \in \mathbb{R}$. More generally, as described

\footnotetext{
${ }^{*}$ This research project was supported by grants from the Natural Sciences and Engineering Research Council of Canada (J.-F. Quessy: RGPIN/6854-2019; M. Mesfioui: RGPIN/065362018).
} 
by Whitt (1976), the Integral stochastic ordering on a class $\mathcal{F}$ of measurable functions states that $Y$ dominates $X$, noted $X \preceq \mathcal{F} Y$, if $\mathrm{E}\{\phi(Y)\} \geq \mathrm{E}\{\phi(X)\}$ for all $\phi \in \mathcal{F}$ for which these expectations exist. Among particular cases of interest are the $s$-increasing convex and concave orders based respectively, for $s \in \mathbb{N}$, on the sets $\mathcal{F}_{s-\mathrm{ICX}}=\left\{\phi: \phi^{[i]} \geq 0 \forall i \leq s\right\}$ and $\mathcal{F}_{s-\mathrm{ICV}}=\left\{\phi:(-1)^{i+1} \phi^{[i]} \geq 0 \forall i \leq\right.$ $s\}$, where $\phi^{[i]}=\mathrm{d}^{i} \phi(x) / \mathrm{d} x^{i}$; see Denuit, Lefevre and Shaked (1998) for details. According for instance to Goovaerts et al. (1990) and Hesselager (1996), the $s$-increasing convex ordering is referred to as the stop-loss order of degree $s-1$, while the $s$-increasing concave ordering is the stochastic dominance of degree $s$.

An extensive literature has been developed to formally check for the stochastic dominance of two populations with respect to $s$-increasing convex (concave) stochastic orderings, i.e. testing for the null and alternative hypotheses

$$
\mathbb{H}_{0}: X \preceq_{s-\mathrm{ICX}} Y \text { and } \mathbb{H}_{1}: X \npreceq_{s-\mathrm{ICX}} Y \text {. }
$$

Such tests have been developed by McFadden (1989) for $s=1,2$ and Anderson (1996), Davidson and Duclos (2000) for $s=1,2,3$ using a Kolmogorov-Smirnov statistic. Noting that these procedures compare distributions at a fixed number of arbitrary points, so that the tests may be inconsistent, Barrett and Donald (2003) propose a global statistic computed from the empirical distribution functions and where p-values are approximated using either a multiplier or a bootstrap method. A test for the stochastic dominance of degree $s=2$ has been proposed by Eubank, Schechtman and Yitzhaki (1993) based on a necessary but not sufficient condition. Some extensions of these procedures have also been proposed. For instance, Linton, Maasoumi and Whang (2005) allow for serial dependence and residuals of linear models, while Linton, Song and Whang (2010) propose to base their decision rule on an improved bootstrap method.

Of a particular interest in this work is the stochastic dominance of a random pair $\left(Y_{1}, Y_{2}\right)$ over another pair $\left(X_{1}, X_{2}\right)$. Specifically, $\left(Y_{1}, Y_{2}\right)$ is said to stochastically dominate $\left(X_{1}, X_{2}\right)$ in the positive quadrant dependence order if the components of the former are more likely than those of the latter to take small values simultaneously; this is formally stated as $\mathbb{P}\left(X_{1} \leq x_{1}, X_{2} \leq x_{2}\right) \leq$ $\mathbb{P}\left(Y_{1} \leq x_{1}, Y_{2} \leq x_{2}\right)$ for all $\left(x_{1}, x_{2}\right) \in \mathbb{R}^{2}$. Under a setup of fixed marginals, Yanagimoto and Okamoto (1969) and Tchen (1980), among others, state that a pair $\left(X_{1}, X_{2}\right)$ is stochastically dominated by $\left(Y_{1}, Y_{2}\right)$ with respect to the concordance order if for all $\left(x_{1}, x_{2}\right) \in \mathbb{R}^{2}$,

$$
\mathbb{P}\left(X_{1}>x_{1}, X_{2}>x_{2}\right) \leq \mathbb{P}\left(Y_{1}>x_{1}, Y_{2}>x_{2}\right) .
$$

Because of the assumption of fixed marginals, Equation (1) is equivalent to $\mathbb{P}\left(X_{1} \leq x_{1}, X_{2} \leq x_{2}\right) \leq \mathbb{P}\left(Y_{1} \leq x_{1}, Y_{2} \leq x_{2}\right)$ for all $\left(x_{1}, x_{2}\right) \in \mathbb{R}^{2}$.

The stochastic ordering as defined in (1) is in fact closely related to the copulas underlying the joint distributions of the pairs to be compared. Specifically, according to a celebrated Theorem of Sklar (1959), there exist copulas $C, D:[0,1]^{2} \rightarrow[0,1]$ such that for all $\left(x_{1}, x_{2}\right) \in \mathbb{R}^{2}$,

$$
\mathbb{P}\left(X_{1} \leq x_{1}, X_{2} \leq x_{2}\right)=C\left\{\mathbb{P}\left(X_{1} \leq x_{1}\right), \mathbb{P}\left(X_{2} \leq x_{2}\right)\right\}
$$

and $\mathbb{P}\left(Y_{1} \leq x_{1}, Y_{2} \leq x_{2}\right)=D\left\{\mathbb{P}\left(Y_{1} \leq x_{1}\right), \mathbb{P}\left(Y_{2} \leq x_{2}\right)\right\}$. 
When the marginal distributions of $\left(X_{1}, X_{2}\right)$ and $\left(Y_{1}, Y_{2}\right)$ are continuous, $C$ and $D$ are unique. If in addition $\mathbb{P}\left(X_{1} \leq x_{1}\right)=\mathbb{P}\left(Y_{1} \leq x_{1}\right)$ and $\mathbb{P}\left(X_{2} \leq x_{2}\right)=$ $\mathbb{P}\left(Y_{2} \leq x_{2}\right)$, then the stochastic dominance of $\left(Y_{1}, Y_{2}\right)$ over $\left(X_{1}, X_{2}\right)$ as defined in (1) reduces to the ordering of their respective copulas in the sense that

$$
C\left(u_{1}, u_{2}\right) \leq D\left(u_{1}, u_{2}\right) \text { for all }\left(u_{1}, u_{2}\right) \in[0,1]^{2} .
$$

In other words, the stochastic dominance of pairs is a copula-based notion under the fixed marginals setup.

The stochastic dominance of pairs on a class $\mathcal{F}$ of measurable functions on $\mathbb{R}^{2}$ has been defined by Marshall (1991), for which $\left(Y_{1}, Y_{2}\right)$ is said to dominate $\left(X_{1}, X_{2}\right)$, noted $\left(X_{1}, X_{2}\right) \preceq_{\mathcal{F}}\left(Y_{1}, Y_{2}\right)$, if $\mathrm{E}\left\{\phi\left(Y_{1}, Y_{2}\right)\right\} \geq \mathrm{E}\left\{\phi\left(X_{1}, X_{2}\right)\right\}$ for all $\phi \in \mathcal{F}$ for which these expectations exist. Particular cases are the $\mathbf{s}$ increasing convex and concave orderings as proposed by Denuit, Lefèvre and Mesfioui (1999); the latter are related respectively, for $\mathbf{s}=\left(s_{1}, s_{2}\right) \in \mathbb{N}^{2}$, to the sets $\mathcal{F}_{\mathbf{s}-\mathrm{ICX}}=\left\{\phi: \phi^{\left[i_{1}, i_{2}\right]} \geq 0 \quad \forall\left(i_{1}, i_{2}\right) \leq \mathbf{s}, i_{1}+i_{2} \geq 1\right\}$ and $\mathcal{F}_{\mathbf{s}-\mathrm{ICV}}=$ $\left\{\phi:(-1)^{i_{1}+i_{2}+1} \phi^{\left[i_{1}, i_{2}\right]} \geq 0 \quad \forall\left(i_{1}, i_{2}\right) \leq \mathbf{s}, i_{1}+i_{2} \geq 1\right\}$, where $\phi^{\left[i_{1}, i_{2}\right]}\left(x_{1}, x_{2}\right)=$ $\partial^{i_{1}+i_{2}} \phi\left(x_{1}, x_{2}\right) / \partial x_{1}^{i_{1}} \partial x_{2}^{i_{2}}$. Note that the stochastic ordering in (1) corresponds to the $(1,1)$-increasing convex ordering.

This paper's starting point is the ascertainment that the s-increasing convex and concave orderings are not copula-based notions, even when $\mathbf{s}=(1,1)$. Indeed, for $\left(X_{1}, X_{2}\right) \preceq_{(1,1)-\mathrm{ICX}}\left(Y_{1}, Y_{2}\right)$ to imply the ordering of their copulas as stated in (2) necessitates that the two pairs belong to a Fréchet class of bivariate distributions having the same marginals. Moreover, as noted by Fernández-Ponce and Rodríguez-Griñolo (2017), the (2,2)-convex and concave orderings of two pairs no longer entail the ordering of their corresponding copulas, even within a Fréchet class, unless additional assumptions are made on the marginals. From our point-of-view, this is a clear limitation of these orders when the prime goal is to gain an insight on the relative strength of dependence between two random couples. This paper has two main goals:

(1) Introduce a family of copula-based stochastic orderings between random pairs (having possibly different marginals) and explore their properties;

(2) Develop a formal statistical methodology to assess the stochastic ordering of two bivariate populations with respect to the new class, in a spirit similar to Barrett and Donald (2003) for the ordering of univariate distributions.

The paper is organized as follows. Section 2 defines the new family of copulabased orderings called s-concordance and explores their properties. Section 3 describes some interpretations of these orders, including the establishment of interesting links with conditional and unconditional versions of Spearman's rank correlation. Section 4 and Section 5 develop a whole statistical methodology to assess the s-concordance ordering of two populations when serial data are available. Section 6 investigates the size and power of the new tests under various data-generating scenarios. Section 7 shows how to adapt these tools to deal with bivariate pairs coming from the same multivariate population, and illustrates the idea on Cook \& Johnson's Uranium exploration dataset; another illustration on 
exchange rate currencies is detailed as well. The proofs are relegated to two appendices and all the code is freely available on www.uqtr.ca/MyMatlabWebpage.

\section{The new class of copula-based orderings}

\subsection{Definition of the s-concordance orders}

The new family of concordance orderings that will be defined is based on the s-increasing convex ordering $\preceq_{\mathbf{s}-\mathrm{ICX}}$ as defined by Denuit, Lefèvre and Mesfioui (1999). Specifically, $\left(X_{1}, X_{2}\right) \preceq_{\mathbf{s}-\mathrm{ICX}}\left(Y_{1}, Y_{2}\right)$ if $\mathrm{E}\left\{\phi\left(X_{1}, X_{2}\right)\right\} \leq \mathrm{E}\left\{\phi\left(Y_{1}, Y_{2}\right)\right\}$ for all $\phi \in \mathcal{F}_{\mathbf{s}-\mathrm{ICX}}=\left\{\phi: \phi^{\left[i_{1}, i_{2}\right]} \geq 0 \quad \forall\left(i_{1}, i_{2}\right) \leq \mathbf{s}, i_{1}+i_{2} \geq 1\right\}$.

Now let $\left(X_{1}, X_{2}\right)$ be a random pair from a distribution function with continuous marginal distributions $F_{1}\left(x_{1}\right)=\mathbb{P}\left(X_{1} \leq x_{1}\right)$ and $F_{2}\left(x_{2}\right)=\mathbb{P}\left(X_{2} \leq x_{2}\right)$, and unique copula $C$ such that $C\left(u_{1}, u_{2}\right)=\mathbb{P}\left\{X_{1} \leq F_{1}^{-1}\left(u_{1}\right), X_{2} \leq F_{2}^{-1}\left(u_{2}\right)\right\}$. Consider another pair $\left(Y_{1}, Y_{2}\right)$ with continuous marginals $G_{1}, G_{2}$ and unique copula $D$. Let $\bar{F}_{1}, \bar{F}_{2}, \bar{G}_{1}$ and $\bar{G}_{2}$ be the marginal survival functions of, respectively, $X_{1}, X_{2}, Y_{1}$ and $Y_{2}$. The following definitions of orthant s-concordance orderings are based on the s-increasing convex ordering of uniformized pairs.

Definition 2.1. The pair $\left(Y_{1}, Y_{2}\right)$ is said to dominate $\left(X_{1}, X_{2}\right)$ according to the lower orthant $\mathbf{s}$-concordance ordering, noted $\left(X_{1}, X_{2}\right) \preceq_{\mathbf{s}-\ell o}^{\star}\left(Y_{1}, Y_{2}\right)$, if

$$
\left(\bar{F}_{1}\left(X_{1}\right), \bar{F}_{2}\left(X_{2}\right)\right) \preceq_{\mathbf{s}-\mathrm{ICX}}\left(\bar{G}_{1}\left(Y_{1}\right), \bar{G}_{2}\left(Y_{2}\right)\right) .
$$

Similarly, the pair $\left(Y_{1}, Y_{2}\right)$ stochastically dominates $\left(X_{1}, X_{2}\right)$ according to the upper orthant $\mathbf{s}$-concordance ordering, noted $\left(X_{1}, X_{2}\right) \preceq_{\mathbf{s}-u o}^{\star}\left(Y_{1}, Y_{2}\right)$, if

$$
\left(F_{1}\left(X_{1}\right), F_{2}\left(X_{2}\right)\right) \preceq_{\mathbf{s}-\mathrm{ICX}}\left(G_{1}\left(Y_{1}\right), G_{2}\left(Y_{2}\right)\right) .
$$

The lower orthant s-concordance ordering could as well be defined in terms of the s-increasing concave ordering $\preceq_{\mathbf{s}-\mathrm{ICV}}$ as described by Denuit, Lefèvre and Mesfioui (1999). Specifically, because of the duality relationship between the s-increasing concave and convex orders expressed in their Proposition 2.3, one has that $\left(X_{1}, X_{2}\right) \preceq_{\mathbf{s}-\ell o}^{\star}\left(Y_{1}, Y_{2}\right)$ could have been defined similarly as $\left(G_{1}\left(Y_{1}\right), G_{2}\left(Y_{2}\right)\right) \preceq_{\text {s-ICV }}\left(F_{1}\left(X_{1}\right), F_{2}\left(X_{2}\right)\right)$.

By construction, the new orthant s-concordance orderings are marginal-free since they depend only on the copula of the pairs being compared. Indeed, for an arbitrary pair $\left(X_{1}, X_{2}\right)$ from a joint distribution with continuous marginals $F_{1}$ and $F_{2}$, it is well known that $\left(F_{1}\left(X_{1}\right), F_{2}\left(X_{2}\right)\right) \sim C$; also, $\left(\bar{F}_{1}\left(X_{1}\right), \bar{F}_{2}\left(X_{2}\right)\right) \sim \widehat{C}$, where $\widehat{C}\left(u_{1}, u_{2}\right)=u_{1}+u_{2}-1+C\left(1-u_{1}, 1-u_{2}\right)$ is the survival copula of $C$. This is to be contrasted with the s-increasing convex ordering that imply some ordering of the marginals. Specifically, if $\left(X_{1}, X_{2}\right) \preceq_{\text {s-ICX }}\left(Y_{1}, Y_{2}\right)$, then $X_{j} \preceq_{s_{j}-\text { ICX }} Y_{j}$ for $j=1,2$, thus involving the marginal distributions.

From the characterization of the (1,1)-increasing convex order, one deduces that $\left(X_{1}, X_{2}\right) \preceq_{(1,1)-\ell o}^{\star}\left(Y_{1}, Y_{2}\right)$ is equivalent to $\mathbb{P}\left\{\bar{F}\left(X_{1}\right)>u_{1}, \bar{F}\left(X_{2}\right)>u_{2}\right\} \leq$ $\mathbb{P}\left\{\bar{G}\left(Y_{1}\right)>u_{1}, \bar{G}\left(Y_{2}\right)>u_{2}\right\}$ for all $\left(u_{1}, u_{2}\right) \in[0,1]^{2}$. Because $\mathbb{P}\left\{\bar{F}\left(X_{1}\right)>\right.$ $\left.u_{1}, \bar{F}\left(X_{2}\right)>u_{2}\right\}=\mathbb{P}\left\{F\left(X_{1}\right)<1-u_{1}, F\left(X_{2}\right)<1-u_{2}\right\}=C\left(1-u_{1}, 1-u_{2}\right)$, 
and similarly $\mathbb{P}\left\{\bar{G}\left(Y_{1}\right)>u_{1}, \bar{G}\left(Y_{2}\right)>u_{2}\right\}=D\left(1-u_{1}, 1-u_{2}\right)$, the lower orthant $(1,1)$-concordance ordering corresponds to the usual concordance ordering; the exact same conclusion applies to the upper orthant $(1,1)$-concordance ordering.

As reported by Fernández-Ponce and Rodríguez-Griñolo (2017), the $(2,2)$ increasing convex ordering $\left(X_{1}, X_{2}\right) \preceq_{(2,2)-\mathrm{ICX}}\left(Y_{1}, Y_{2}\right)$ is characterized by

$\int_{t_{1}}^{\infty} \int_{t_{2}}^{\infty} \mathbb{P}\left(X_{1}>x_{1}, X_{2}>x_{2}\right) \mathrm{d} x_{1} \mathrm{~d} x_{2} \leq \int_{t_{1}}^{\infty} \int_{t_{2}}^{\infty} \mathbb{P}\left(Y_{1}>x_{1}, Y_{2}>x_{2}\right) \mathrm{d} x_{1} \mathrm{~d} x_{2}$.

The lower orthant $(2,2)$-concordance ordering $\preceq_{(2,2)-\ell_{o}}^{\star}$ is therefore based on the integration over $\left[t_{1}, 1\right] \times\left[t_{2}, 1\right]$ of $\mathbb{P}\left\{\bar{F}\left(X_{1}\right)>x_{1}, \bar{F}\left(X_{2}\right)>x_{2}\right\}=C\left(1-x_{1}, 1-x_{2}\right)$ and $\mathbb{P}\left\{\bar{G}\left(Y_{1}\right)>x_{1}, \bar{G}\left(Y_{2}\right)>x_{2}\right\}=D\left(1-x_{1}, 1-x_{2}\right)$. Thus, from the simple change-of-variable $u_{1}=1-x_{1}$ and $u_{2}=1-x_{2}$, one has $\left(X_{1}, X_{2}\right) \preceq_{(2,2)-\ell o}^{\star}$ $\left(Y_{1}, Y_{2}\right)$ if and only if, for all $\left(t_{1}, t_{2}\right) \in[0,1]^{2}$,

$$
\int_{0}^{t_{1}} \int_{0}^{t_{2}} C\left(u_{1}, u_{2}\right) \mathrm{d} u_{1} \mathrm{~d} u_{2} \leq \int_{0}^{t_{1}} \int_{0}^{t_{2}} D\left(u_{1}, u_{2}\right) \mathrm{d} u_{1} \mathrm{~d} u_{2} .
$$

Similarly, $\left(X_{1}, X_{2}\right) \preceq_{(2,2)-u o}^{\star}\left(Y_{1}, Y_{2}\right)$ if and only if, for all $\left(t_{1}, t_{2}\right) \in[0,1]^{2}$,

$$
\int_{t_{1}}^{1} \int_{t_{2}}^{1} C\left(u_{1}, u_{2}\right) \mathrm{d} u_{1} \mathrm{~d} u_{2} \leq \int_{t_{1}}^{1} \int_{t_{2}}^{1} D\left(u_{1}, u_{2}\right) \mathrm{d} u_{1} \mathrm{~d} u_{2} .
$$

Generally speaking, the lower orthant s-concordance ordering compares the dependence structures of the pairs on the lower orthants of $[0,1]^{2}$, while its upper orthant version does it on the upper orthants of $[0,1]^{2}$.

\subsection{Basic properties}

The first result concerns the duality that exists between $\preceq_{\mathbf{s}-\ell o}^{\star}$ and $\preceq_{\mathbf{s}-u o}^{\star}$.

Property 2.1. $\left(X_{1}, X_{2}\right) \preceq_{\mathbf{s}-u o}^{\star}\left(Y_{1}, Y_{2}\right) \Longleftrightarrow\left(-X_{1},-X_{2}\right) \preceq_{\mathbf{s}-\ell o}^{\star}\left(-Y_{1},-Y_{2}\right)$.

As stated next, the new class of orderings is hierarchical.

Property 2.2. If $\left(X_{1}, X_{2}\right) \preceq_{\mathbf{s}-\ell o}^{\star}\left(Y_{1}, Y_{2}\right)$ for some $\mathbf{s} \in \mathbb{N}^{2}$, then $\left(X_{1}, X_{2}\right) \preceq_{\mathbf{s}^{\prime}-\ell o}^{\star}$ $\left(Y_{1}, Y_{2}\right)$ for all $\mathbf{s}^{\prime} \geq \mathbf{s}$. Similarly, $\left(X_{1}, X_{2}\right) \preceq_{\mathbf{s}-u o}^{\star}\left(Y_{1}, Y_{2}\right)$ entails $\left(X_{1}, X_{2}\right) \preceq_{\mathbf{s}^{\prime}-u o}^{\star}$ $\left(Y_{1}, Y_{2}\right)$ for all $\mathbf{s}^{\prime} \geq \mathbf{s}$.

Since copulas are invariant under monotone increasing transformations of the marginals, it is expected that the lower and upper orthant s-concordance orderings be invariant under such mappings. The next result establishes this basic fact, and explores situations involving monotone decreasing transformations.

Property 2.3. Let $\preceq_{\mathbf{s}}^{\star}$ be either $\preceq_{\mathbf{s}-\ell_{o}}^{\star}$ or $\preceq_{\mathbf{s}-u o}^{\star}$. For $\psi_{1}, \psi_{2}: \mathbb{R} \rightarrow \mathbb{R}$ strictly monotone, one has $\left(\psi_{1}\left(X_{1}\right), \psi_{2}\left(X_{2}\right)\right) \preceq_{\mathbf{s}}^{\star}\left(\psi_{1}\left(Y_{1}\right), \psi_{2}\left(Y_{2}\right)\right)$ if and only if

(i) $\left(X_{1}, X_{2}\right) \preceq_{\mathbf{s}}^{\star}\left(Y_{1}, Y_{2}\right)$, if both $\psi_{1}$ and $\psi_{2}$ are increasing;

(ii) $\left(-X_{1},-X_{2}\right) \preceq_{\mathrm{s}}^{\star}\left(-Y_{1},-Y_{2}\right)$, if both $\psi_{1}$ and $\psi_{2}$ are decreasing; 
(iii) $\left(X_{1},-X_{2}\right) \preceq_{\mathbf{s}}^{\star}\left(Y_{1},-Y_{2}\right)$, if $\psi_{1}$ is increasing and $\psi_{2}$ is decreasing;

(iv) $\left(-X_{1}, X_{2}\right) \preceq_{\mathrm{s}}^{\star}\left(-Y_{1}, Y_{2}\right)$, if $\psi_{1}$ is decreasing and $\psi_{2}$ is increasing.

When $C=\widehat{C}$, where $\widehat{C}$ is the survival copula of $C$, it is said that $C$ is a radially symmetric copula. In generic terms, radial symmetry means that the lower tail of (the density of) $C$ has the same form as the upper tail. Knowing that, the next result which establishes the equivalence between $\preceq_{\mathbf{s}-\ell_{o}}^{\star}$ and $\preceq_{\mathbf{s}-u o}^{\star}$ under radially symmetric dependence structures will come as no surprise.

Property 2.4. If the respective copulas of $\left(X_{1}, X_{2}\right)$ and $\left(Y_{1}, Y_{2}\right)$ are radially symmetric, then $\left(X_{1}, X_{2}\right) \preceq_{\mathbf{s}-\ell o}^{\star}\left(Y_{1}, Y_{2}\right) \Longleftrightarrow\left(X_{1}, X_{2}\right) \preceq_{\mathbf{s}-u o}^{\star}\left(Y_{1}, Y_{2}\right)$.

\subsection{The fixed marginals setup and beyond}

Suppose that the pairs $\left(X_{1}, X_{2}\right)$ and $\left(Y_{1}, Y_{2}\right)$ belong to the same Fréchet class of bivariate distributions with marginals $F_{1}$ and $F_{2}$. In that case, the lower and upper orthant $(1,1)$-concordance orderings are equivalent to the $(1,1)$ increasing convex order. Things are not as straightforward when $\mathbf{s} \neq(1,1)$. As one can deduce from Theorem 3 of Fernández-Ponce and Rodríguez-Griñolo (2017), $\left(X_{1}, X_{2}\right) \preceq_{(2,2)-u o}^{\star}\left(Y_{1}, Y_{2}\right)$ entails $\left(X_{1}, X_{2}\right) \preceq_{(2,2)-\operatorname{ICX}}\left(Y_{1}, Y_{2}\right)$ not only if the pairs belong to the same Fréchet class, but if in addition $F_{1}, F_{2}$ have decreasing densities. On the other side, a consequence of Theorem 4 of these authors is that $\left(X_{1}, X_{2}\right) \preceq_{(2,2)-\mathrm{ICX}}\left(Y_{1}, Y_{2}\right)$ entails $\left(X_{1}, X_{2}\right) \preceq_{(2,2)-u o}^{\star}\left(Y_{1}, Y_{2}\right)$ if $F_{1}, F_{2}$ have increasing densities. One then deduces that the only case where $\preceq_{(2,2)-u o}^{\star}$ and $\preceq_{(2,2)-\text { ICX }}$ are equivalent is under uniform marginal distributions.

The next result generalizes these findings by Fernández-Ponce and RodríguezGriñolo (2017) to an arbitrary $\mathbf{s}=\left(s_{1}, s_{2}\right) \in \mathbb{N}^{2}$. Before stating it, a real-valued function $\phi$ is said to be $s$-convex if $\phi \in \mathcal{F}_{s-\mathrm{ICX}}=\left\{\phi: \phi^{[i]} \geq 0 \forall i \leq s\right\}$, and $s$ concave if $\phi \in \mathcal{F}_{s-\mathrm{ICV}}=\left\{\phi:(-1)^{i+1} \phi^{[i]} \geq 0 \forall i \leq s\right\}$, where $\phi^{[i]}=\overline{\mathrm{d}}^{i} \phi(x) / \mathrm{d} x^{i}$.

Proposition 2.1. Let $\left(X_{1}, X_{2}\right)$ and $\left(Y_{1}, Y_{2}\right)$ be in the same Fréchet class of bivariate distributions with continuous marginals $F_{1}$ and $F_{2}$.

Lower orthant s-concordance

(i) If $F_{1}^{-1}$ is $s_{1}$-concave and $F_{2}^{-1}$ is $s_{2}$-concave, then $\left(X_{1}, X_{2}\right) \preceq_{\mathbf{s}-\ell o}^{\star}\left(Y_{1}, Y_{2}\right)$ entails $\left(-X_{1},-X_{2}\right) \preceq_{\mathbf{s}-\mathrm{ICX}}\left(-Y_{1},-Y_{2}\right)$.

(ii) If $F_{1}$ is $s_{1}$-concave and $F_{2}$ is $s_{2}$-concave, then $\left(-X_{1},-X_{2}\right) \preceq_{\mathbf{s}-I C X}\left(-Y_{1},-Y_{2}\right)$ entails $\left(X_{1}, X_{2}\right) \preceq_{\mathbf{s}-\ell o}^{\star}\left(Y_{1}, Y_{2}\right)$.

Upper orthant s-concordance

(iii) If $F_{1}^{-1}$ is $s_{1}$-convex and $F_{2}^{-1}$ is $s_{2}$-convex, then $\left(X_{1}, X_{2}\right) \preceq_{\mathbf{s}-u o}^{\star}\left(Y_{1}, Y_{2}\right)$ entails $\left(X_{1}, X_{2}\right) \preceq_{\text {s-ICX }}\left(Y_{1}, Y_{2}\right)$.

(iv) If $F_{1}$ is $s_{1}$-convex and $F_{2}$ is $s_{2}$-convex, then $\left(X_{1}, X_{2}\right) \preceq_{\mathbf{s}-\text { ICX }}\left(Y_{1}, Y_{2}\right)$ entails $\left(X_{1}, X_{2}\right) \preceq_{\mathbf{s}-u o}^{\star}\left(Y_{1}, Y_{2}\right)$.

One recovers Theorem 3 of Fernández-Ponce and Rodríguez-Griñolo (2017) by letting $\mathbf{s}=(2,2)$ in parts (i) and (iii) of Proposition 2.1, since the 2-concavity 
of $F_{1}^{-1}, F_{2}^{-1}$ means that $F_{1}, F_{2}$ have decreasing densities, while their 2-convexity is equivalent to having increasing densities. For similar reasons, their Theorem 4 is a special case of parts (ii) and (iv) of Proposition 2.1.

\section{4. s-concordance orderings of popular copula families}

Consider the Normal, Clayton, Gumbel and Plackett copulas whose expressions are given in Table 1. These models have been extensively used for bivariate copula modeling and their properties are well-known; see Nelsen (2006) and Joe (2015), for instance. For example, the family of Normal copulas share with the Plackett dependence structures the property of radial symmetry.

TABLE 1

The Normal, Clayton, Gumbel and Plackett copulas

\begin{tabular}{|c|c|c|}
\hline Copula & Expression of the copula & Parameter space \\
\hline Normal $^{a}$ & $\Phi_{\theta}\left\{\Phi^{-1}\left(u_{1}\right), \Phi^{-1}\left(u_{2}\right)\right\}$ & $\theta \in[-1,1]$ \\
\hline Clayton & $\left(u_{1}^{-\theta}+u_{2}^{-\theta}-1\right)^{-1 / \theta}$ & $\theta \in(0, \infty)$ \\
\hline Gumbel & $\exp \left\{-\left(\left|\ln u_{1}\right|^{1 /(1-\theta)}+\left|\ln u_{2}\right|^{1 /(1-\theta)}\right)^{1-\theta}\right\}$ & $\theta \in[0,1]$ \\
\hline \multirow{2}{*}{ Plackett $^{b}$} & $g_{\theta}\left(u_{1}, u_{2}\right)-\sqrt{g_{\theta}^{2}\left(u_{1}, u_{2}\right)-8 \theta(\theta+1) u_{1} u_{2}}$ & \multirow{2}{*}{$\theta \in[-1,1]$} \\
\hline & $4 \theta$ & \\
\hline
\end{tabular}

These four copula families are parametrized such that $C_{\theta}\left(u_{1}, u_{2}\right) \leq C_{\theta^{\prime}}\left(u_{1}, u_{2}\right)$ for all $\left(u_{1}, u_{2}\right) \in(0,1)^{2}$ when $\theta \leq \theta^{\prime}$. In view of Property 2.2, they are also ordered with respect to $\mathbf{s}$-concordance for any $\mathbf{s} \in \mathbb{N}^{2}$. Things become less clear, and in fact more interesting, when the goal is to stochastically compare two copulas $C$ and $D$ that belong to different parametric families. To this end, let $\left(X_{1}, X_{2}\right)$ and $\left(Y_{1}, Y_{2}\right)$ be random pairs with copulas $C$ and $D$, respectively. Table 2 reports scenarios when $\left(X_{1}, X_{2}\right)$ and $\left(Y_{1}, Y_{2}\right)$ cannot be ordered according to the usual concordance, i.e. $\left(X_{1}, X_{2}\right) \npreceq_{(1,1)-\ell_{o}}^{\star}\left(Y_{1}, Y_{2}\right)$, while $\left(X_{1}, X_{2}\right) \preceq_{(2,2)-\ell o}^{\star}\left(Y_{1}, Y_{2}\right)$. In order to standardize the comparisons, each model has been parametrized in terms of its associated Kendall's tau, i.e.

$$
\tau_{C}=4 \int_{0}^{1} \int_{0}^{1} C\left(u_{1}, u_{2}\right) \mathrm{d} C\left(u_{1}, u_{2}\right)-1 .
$$

As an example of a situation where $\npreceq_{(1,1)-\ell_{0}}^{\star}$ and $\preceq_{(2,2)-\ell o}^{\star}$, consider $D$ being the Clayton copula with $\tau_{D}=1 / 3$; in that case, $\left(X_{1}, X_{2}\right) \npreceq_{(1,1)-\ell o}^{\star}\left(Y_{1}, Y_{2}\right)$ and 
TABLE 2

Some scenarios where the respective copulas $C$ and $D$ of random pairs $\left(X_{1}, X_{2}\right)$ and

$\left(Y_{1}, Y_{2}\right)$ are such that $\left(X_{1}, X_{2}\right){\aleph_{(1,1)-\ell o}^{\star}}^{\star}\left(Y_{1}, Y_{2}\right)$ and $\left(X_{1}, X_{2}\right) \preceq_{(2,2)-\ell o}^{\star}\left(Y_{1}, Y_{2}\right)$

\begin{tabular}{ccc||c|c|c}
\hline$C$ & $D$ & $\tau_{D}$ & $\preceq_{(1,1)-\ell o}^{\star}$ & $\preceq_{(2,2)-\ell o}^{\star}$ & $\varlimsup_{(1,1)-\ell o}^{\star}$ and $\preceq_{(2,2)-\ell o}^{\star}$ \\
\hline \multirow{2}{*}{ Gumbel } & Clayton & $1 / 3$ & $\tau_{C} \in[0, .0071]$ & $\tau_{C} \in[0, .3346]$ & $\tau_{C} \in(.0071, .3346]$ \\
& & $2 / 3$ & $\tau_{C} \in[0, .0281]$ & $\tau_{C} \in[0, .6640]$ & $\tau_{C} \in(.0281, .6640]$ \\
& & & & \\
Plackett & Clayton & $1 / 3$ & $\tau_{C} \in[0, .1556]$ & $\tau_{C} \in[0, .3330]$ & $\tau_{C} \in(.1556, .3330]$ \\
& & $2 / 3$ & $\tau_{C} \in[0, .3536]$ & $\tau_{C} \in[0, .6750]$ & $\tau_{C} \in(.3536, .6750]$ \\
& & & & \\
Gumbel & Plackett & $1 / 3$ & $\tau_{C} \in[0, .0250]$ & $\tau_{C} \in[0, .2529]$ & $\tau_{C} \in(.0250, .2529]$ \\
& & $2 / 3$ & $\tau_{C} \in[0, .1568]$ & $\tau_{C} \in[0, .5825]$ & $\tau_{C} \in(.1568, .5825]$ \\
\hline
\end{tabular}

$\left(X_{1}, X_{2}\right) \preceq_{(2,2)-\ell o}^{\star}\left(Y_{1}, Y_{2}\right)$ if the copula $C$ of $\left(X_{1}, X_{2}\right)$ belongs to the Gumbel family with $\tau_{C} \in(.0071, .3346]$. It also happens when $\tau_{D}=2 / 3$ and $\tau_{C} \in$ $(.0281, .6640]$, and more particularly when $\tau_{C}=\tau_{D}=1 / 3$. Another example occurs when $D$ is the Clayton copula with $\tau_{D}=1 / 3$ and $C$ belongs to the Plackett family with $\tau_{C} \in(.1556, .3330]$.

\subsection{Characterization of $\preceq_{\mathrm{s}-\ell o}^{\star}$ and $\preceq_{\mathrm{s}-u o}^{\star}$}

A characterization of the s-increasing convex ordering of random pair was deduced by Denuit, Lefèvre and Mesfioui (2003) in terms of iterated distributions. Such characterizations for the s-concordance orders $\preceq_{\mathbf{s}-\ell_{0}}^{\star}$ and $\preceq_{\mathbf{s}-u o}^{\star}$ are developed here. The latter will prove useful later to formally test for s-concordance ordering. To this end, let $\ell^{\infty}\left([0,1]^{2}\right)$ be the space of bounded functions on $[0,1]^{2}$. For $g \in \ell^{\infty}\left([0,1]^{2}\right)$, define for each $\mathbf{i} \in \mathbb{N}^{2}$ the operator $\mathcal{J}_{\mathbf{i}}: \ell^{\infty}\left([0,1]^{2}\right) \rightarrow$ $\ell^{\infty}\left([0,1]^{2}\right)$ such that for $\left(u_{1}, u_{2}\right) \in[0,1]^{2}, \mathcal{J}_{(1,1)}\left(u_{1}, u_{2} ; g\right)=g\left(u_{1}, u_{2}\right)$ and for $\mathbf{i} \in \mathbb{N}^{2}$ with $\max \left(i_{1}, i_{2}\right)>1$, one has recursively

$$
\begin{aligned}
\mathcal{J}_{\mathbf{i}}\left(u_{1}, u_{2} ; g\right) & =\int_{0}^{u_{1}} \mathcal{J}_{\left(i_{1}-1, i_{2}\right)}\left(x_{1}, u_{2} ; g\right) \mathrm{d} x_{1} \quad\left(\text { if } i_{1}>1\right) \\
& =\int_{0}^{u_{2}} \mathcal{J}_{\left(i_{1}, i_{2}-1\right)}\left(u_{1}, x_{2} ; g\right) \mathrm{d} x_{2} \quad\left(\text { if } i_{2}>1\right) .
\end{aligned}
$$

As formally stated in the following result, the lower orthant s-concordance ordering of random pairs can be seen as a functional of the difference between their respectively copulas. Similarly, the upper orthant s-concordance ordering appears as a functional of their associated survival copulas.

Proposition 2.2. Let $\left(X_{1}, X_{2}\right)$ and $\left(Y_{1}, Y_{2}\right)$ be random pairs with continuous marginals and respective copulas $C$ and $D$. For $\mathcal{E}_{\mathbf{s}}=\{\mathbf{s}\} \cup\left\{\mathbf{i} \in \mathbb{N}^{2}: \mathbf{i} \leq\right.$ $\left.\mathbf{s}, \min \left(i_{1}, i_{2}\right)>1\right\}$, one has $\left(X_{1}, X_{2}\right) \preceq_{\mathbf{s}-\ell_{o}}^{\star}\left(Y_{1}, Y_{2}\right)$ if and only if

$$
\max _{\mathbf{i} \in \mathcal{E}_{\mathbf{s}}} \mathcal{J}_{\mathbf{i}}\left(u_{1}^{\mathbb{I}\left(i_{1}=s_{1}\right)}, u_{2}^{\mathbb{I}\left(i_{2}=s_{2}\right)} ; C-D\right) \leq 0 \quad \text { for all }\left(u_{1}, u_{2}\right) \in[0,1]^{2} .
$$


Also, $\left(X_{1}, X_{2}\right) \preceq_{\mathbf{s}-u o}^{\star}\left(Y_{1}, Y_{2}\right)$ if and only if

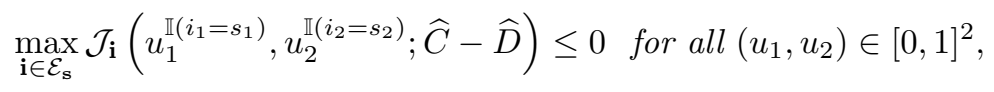

where $\widehat{C}$ (resp. $\widehat{D}$ ) is the survival copula of $C$ (resp. D).

There is only one condition needed to establish the s-concordance ordering of two pairs when $\mathbf{s} \leq(2,2)$, since $\mathcal{E}_{\mathbf{s}}=\{\mathbf{s}\}$ in that case. Proposition $(2.2)$ then reduces to $\left(X_{1}, X_{2}\right) \preceq_{\mathbf{s}-\ell o}^{\star}\left(Y_{1}, Y_{2}\right)$ if and only if

$$
\mathcal{J}_{\mathbf{s}}\left(u_{1}, u_{2} ; C-D\right) \leq 0 \text { for all }\left(u_{1}, u_{2}\right) \in[0,1]^{2} .
$$

In particular, $\left(X_{1}, X_{2}\right) \preceq_{(1,1)-\ell_{o}}^{\star}\left(Y_{1}, Y_{2}\right)$ if and only if $C\left(u_{1}, u_{2}\right) \leq D\left(u_{1}, u_{2}\right)$ for all $\left(u_{1}, u_{2}\right) \in[0,1]^{2}$, while $\left(X_{1}, X_{2}\right) \preceq_{(2,2)-\ell o}^{\star}\left(Y_{1}, Y_{2}\right)$ if and only if Equation (3)

holds. Since $\widehat{C}\left(x_{1}, x_{2}\right)-\widehat{D}\left(x_{1}, x_{2}\right)=C\left(1-x_{1}, 1-x_{2}\right)-D\left(1-x_{1}, 1-x_{2}\right)$, the simple change of variable $y_{1}=1-x_{1}$ and $y_{2}=1-x_{2}$ allows to conclude that $\left(X_{1}, X_{2}\right) \preceq_{(2,2)-u o}^{\star}\left(Y_{1}, Y_{2}\right)$ if and only if Equation (4) holds.

\section{Some interpretations of the new concordance orders}

\subsection{The $(2,1)$ and $(1,2)$-concordance orderings}

As outlined by Denuit and Mesfioui (2017), the $(2,1)$-increasing concave ordering $\left(Y_{1}, Y_{2}\right) \preceq_{(2,1)-\mathrm{ICV}}\left(X_{1}, X_{2}\right)$ holds if and only if for all $\left(t_{1}, t_{2}\right) \in \mathbb{R}^{2}$,

(i) $\bar{F}_{2}\left(t_{2}\right) \geq \bar{G}_{2}\left(t_{2}\right)$;

(ii) $\mathrm{E}\left\{\left(t_{1}-X_{1}\right)_{+} \mathbb{I}\left(X_{2} \leq t_{2}\right)\right\} \leq \mathrm{E}\left\{\left(t_{1}-Y_{1}\right)_{+} \mathbb{I}\left(Y_{2} \leq t_{2}\right)\right\}$.

While condition (i) expresses the usual stochastic dominance of $X_{2}$ over $Y_{2}$, the second condition compares the strength of the corresponding conditional shortfalls of the pairs. Specifically, $\left(t_{1}-X_{1}\right)_{+} \mathbb{I}\left(X_{2} \leq t_{2}\right)$ vanishes given that $X_{2}$ is larger than the threshold $t_{2}$, so that the shortfall $\left(t_{1}-X_{1}\right)_{+}$with respect to the threshold $t_{1}$ becomes irrelevant. This shows some sort of compensation between the components of $\left(t_{1}-X_{1}\right)_{+} \mathbb{I}\left(X_{2} \leq t_{2}\right)$. Also, as shown in Proposition 3.2 of Denuit and Mesfioui (2017), the $(2,1)$-concave order characterizes the Rothschild-Stiglitz type of increase in risk as introduced by Guo et al. (2016).

As was noted after the statement of Definition 2.1, the lower orthant sconcordance $\left(X_{1}, X_{2}\right) \preceq_{\mathbf{s}-\ell_{o}}^{\star}\left(Y_{1}, Y_{2}\right)$ holds if and only if $\left(G_{1}\left(Y_{1}\right), G_{2}\left(Y_{2}\right)\right) \preceq_{\mathbf{s}-\mathrm{ICV}}$ $\left(F_{1}\left(X_{1}\right), F_{2}\left(X_{2}\right)\right)$. Since the marginal distributions of the pairs to be compared are, by construction, uniform on $(0,1)$, condition (i) becomes irrelevant and the lower orthant $(2,1)$-concordance $\left(X_{1}, X_{2}\right) \preceq_{(2,1)-\ell o}^{\star}\left(Y_{1}, Y_{2}\right)$ is equivalent to

$$
\mathrm{E}\left\{\left(t_{1}-F_{1}\left(X_{1}\right)\right)_{+} \mathbb{I}\left(F_{2}\left(X_{2}\right) \leq t_{2}\right)\right\} \leq \mathrm{E}\left\{\left(t_{1}-G_{1}\left(Y_{1}\right)\right)_{+} \mathbb{I}\left(G_{2}\left(Y_{2}\right) \leq t_{2}\right)\right\} .
$$

Clearly, the above inequality holds when the components of the pair $\left(Y_{1}, Y_{2}\right)$ are more associated than those of $\left(X_{1}, X_{2}\right)$, since then, $\left(t_{1}-F_{1}\left(X_{1}\right)\right)_{+} \mathbb{I}\left(F_{2}\left(X_{2}\right) \leq t_{2}\right)$ tends to vanish more frequently than $\left(t_{1}-G_{1}\left(Y_{1}\right)\right)_{+} \mathbb{I}\left(G_{2}\left(Y_{2}\right) \leq t_{2}\right)$. Otherwise, similar interpretations as those above can be made, but at the level of the dependence structures of the pairs that are being compared. 


\subsection{Consequences of $\preceq_{\mathrm{s}-\ell o}^{\star}$ on Spearman's rho and other concordance measures}

Several measures of dependence are concordance measures in the sense given by Scarsini (1984); see also Nelsen (2002). Generally, they can be expressed in terms of the concordance operator between two copulas as defined by

$$
Q\left(C_{1}, C_{2}\right)=\mathbb{P}\left(\mathbf{U}_{1}<\mathbf{U}_{2}\right)+\mathbb{P}\left(\mathbf{U}_{1}>\mathbf{U}_{2}\right)=2 \mathbb{P}\left(\mathbf{U}_{1}<\mathbf{U}_{2}\right),
$$

where $\mathbf{U}_{1} \sim C_{1}$ and $\mathbf{U}_{2} \sim C_{2}$ are independent pairs. For example, the Kendall and Spearman measures of dependence of a random pair $\left(X_{1}, X_{2}\right)$ with copula $C$ can be expressed respectively as

$$
\begin{aligned}
\tau\left(X_{1}, X_{2}\right) & =\frac{Q(C, C)-Q(\Pi, \Pi)}{Q(M, M)-Q(\Pi, \Pi)}=4 \int_{0}^{1} \int_{0}^{1} C\left(u_{1}, u_{2}\right) \mathrm{d} C\left(u_{1}, u_{2}\right)-1 \\
\text { and } \rho^{\mathrm{Sp}}\left(X_{1}, X_{2}\right) & =\frac{Q(C, \Pi)-Q(\Pi, \Pi)}{Q(M, \Pi)-Q(\Pi, \Pi)}=12 \int_{0}^{1} \int_{0}^{1} C\left(u_{1}, u_{2}\right) \mathrm{d} u_{1} \mathrm{~d} u_{2}-3,
\end{aligned}
$$

where $\Pi\left(u_{1}, u_{2}\right)=u_{1} u_{2}$ and $M\left(u_{1}, u_{2}\right)=\min \left(u_{1}, u_{2}\right)$ are the copulas of independence and perfect positive dependence, respectively. In fact, concordance measures are closely linked to the concordance ordering $\preceq_{(1,1)-\ell 0^{\star}}^{*}$ On one side, it can be shown that if $C_{1} \leq C_{1}^{\star}$ and $C_{2} \leq C_{2}^{\star}$, then $Q\left(C_{1}, C_{2}\right) \leq Q\left(C_{1}^{\star}, C_{2}^{\star}\right)$. On the other side, $C \leq D$ when $\left(X_{1}, X_{2}\right) \preceq_{(1,1)-\ell_{o}}^{\star}\left(Y_{1}, Y_{2}\right)$, so that $\gamma\left(X_{1}, X_{2}\right) \leq$ $\gamma\left(Y_{1}, Y_{2}\right)$ for any concordance measure $\gamma$. In particular, $\tau\left(X_{1}, X_{2}\right) \leq \tau\left(Y_{1}, Y_{2}\right)$ and $\rho^{\mathrm{Sp}}\left(X_{1}, X_{2}\right) \leq \rho^{\mathrm{Sp}}\left(Y_{1}, Y_{2}\right)$ when $\left(X_{1}, X_{2}\right) \preceq_{(1,1)-\ell_{o}}^{\star}\left(Y_{1}, Y_{2}\right)$.

Note that if $\left(X_{1}, X_{2}\right) \preceq_{(1,1)-\ell_{0}}^{\star}\left(Y_{1}, Y_{2}\right)$ holds strictly, i.e. $C \neq D$ a.s., then $\rho^{\mathrm{Sp}}\left(X_{1}, X_{2}\right)<\rho^{\mathrm{Sp}}\left(Y_{1}, Y_{2}\right)$, i.e. $\rho^{\mathrm{Sp}}\left(X_{1}, X_{2}\right) \neq \rho^{\mathrm{Sp}}\left(Y_{1}, Y_{2}\right)$. The $(2,2)-$ concordance ordering imposes a milder restriction, since $\left(X_{1}, X_{2}\right) \preceq_{(2,2)-\ell o}^{\star}$ $\left(Y_{1}, Y_{2}\right)$ may happen to hold strictly while $\rho^{\mathrm{Sp}}\left(X_{1}, X_{2}\right)=\rho^{\mathrm{Sp}}\left(Y_{1}, Y_{2}\right)$.

\subsection{Consequences of $\preceq_{(2,2)-\ell o}^{\star}$ and $\preceq_{(2,2)-u o}^{\star}$ on conditional versions of Spearman's rho}

As noted in (3), $\left(X_{1}, X_{2}\right) \preceq_{(2,2)-\ell o}^{\star}\left(Y_{1}, Y_{2}\right)$ entails the ordering of the lower orthant integrated copulas; in view of $(4),\left(X_{1}, X_{2}\right) \preceq_{(2,2)-u o}^{\star}\left(Y_{1}, Y_{2}\right)$ implies a similar ordering, but with respect to upper orthant integration. In particular,

$$
\int_{0}^{1} \int_{0}^{1} C\left(x_{1}, x_{2}\right) \mathrm{d} x_{2} \mathrm{~d} x_{1} \leq \int_{0}^{1} \int_{0}^{1} D\left(x_{1}, x_{2}\right) \mathrm{d} x_{2} \mathrm{~d} x_{1} .
$$

In fact, this inequality holds when $\left(X_{1}, X_{2}\right) \preceq_{\mathbf{s}-\ell_{o}}^{\star}\left(Y_{1}, Y_{2}\right)$ for any $\mathbf{s} \in \mathbb{N}^{2}$. This easily entails $\rho^{\mathrm{Sp}}\left(X_{1}, X_{2}\right) \leq \rho^{\mathrm{Sp}}\left(Y_{1}, Y_{2}\right)$, so that the s-concordance ordering of two pairs always implies the ordering of their corresponding Spearman's rho. But still more can be said about Spearman's rho under the $(2,2)$-concordance 
ordering. To this end, first define a version of the concordance operator in (7) constrained to the lower rectangle $\left[0, u_{1}\right] \times\left[0, u_{2}\right]$, namely

$$
Q_{\mathbf{u}}\left(C_{1}, C_{2}\right)=2 \mathbb{P}\left(\mathbf{U}_{1}<\mathbf{U}_{2} \cap \mathbf{U}_{2}<\mathbf{u}\right), \quad \mathbf{u}=\left(u_{1}, u_{2}\right) .
$$

Replacing the concordance operator $Q$ by $Q_{\mathbf{u}}$ in the definitions of Kendall and Spearman measures of association yields conditional versions of these concordance measures. Doing so for Spearman's rho, one obtains

$$
\rho_{u_{1}, u_{2}}^{\mathrm{Sp}}\left(X_{1}, X_{2}\right)=\frac{\int_{0}^{u_{1}} \int_{0}^{u_{2}} C\left(x_{1}, x_{2}\right) \mathrm{d} x_{1} \mathrm{~d} x_{2}-\int_{0}^{u_{1}} \int_{0}^{u_{2}} \Pi\left(x_{1}, x_{2}\right) \mathrm{d} x_{1} \mathrm{~d} x_{2}}{\int_{0}^{u_{1}} \int_{0}^{u_{2}} M\left(x_{1}, x_{2}\right) \mathrm{d} x_{1} \mathrm{~d} x_{2}-\int_{0}^{u_{1}} \int_{0}^{u_{2}} \Pi\left(x_{1}, x_{2}\right) \mathrm{d} x_{1} \mathrm{~d} x_{2}} .
$$

This is exactly the bivariate version of the conditional Spearman's rho as defined by Schmid and Schmidt (2007) while letting $d=2$ and $g:=\mathbb{I}\left(\cdot \leq u_{1}, \cdot \leq u_{2}\right)$ in their Equation (4). As a consequence, $\left(X_{1}, X_{2}\right) \preceq_{(2,2)-\ell_{o}}^{\star}\left(Y_{1}, Y_{2}\right)$ if and only if $\rho_{u_{1}, u_{2}}^{\mathrm{Sp}}\left(X_{1}, X_{2}\right) \leq \rho_{u_{1}, u_{2}}^{\mathrm{Sp}}\left(Y_{1}, Y_{2}\right)$ for all $\left(u_{1}, u_{2}\right) \in[0,1]^{2}$.

The upper-orthant $(2,2)$-concordance also entails the ordering of an "upper orthant" version $\widetilde{\rho}_{u_{1}, u_{2}}^{\mathrm{Sp}}\left(X_{1}, X_{2}\right)$ of $\rho_{u_{1}, u_{2}}^{\mathrm{Sp}}\left(X_{1}, X_{2}\right)$ that arises by integrating on $\left[u_{1}, 1\right] \times\left[u_{2}, 1\right]$ instead of $\left[0, u_{1}\right] \times\left[0, u_{2}\right]$, namely

$$
\tilde{\rho}_{u_{1}, u_{2}}^{\mathrm{Sp}}\left(X_{1}, X_{2}\right)=\frac{\int_{u_{1}}^{1} \int_{u_{2}}^{1} C\left(x_{1}, x_{2}\right) \mathrm{d} x_{1} \mathrm{~d} x_{2}-\int_{u_{1}}^{1} \int_{u_{2}}^{1} \Pi\left(x_{1}, x_{2}\right) \mathrm{d} x_{1} \mathrm{~d} x_{2}}{\int_{u_{1}}^{1} \int_{u_{2}}^{1} M\left(x_{1}, x_{2}\right) \mathrm{d} x_{1} \mathrm{~d} x_{2}-\int_{u_{1}}^{1} \int_{u_{2}}^{1} \Pi\left(x_{1}, x_{2}\right) \mathrm{d} x_{1} \mathrm{~d} x_{2}} .
$$

Hence, based on (4), one can conclude that $\left(X_{1}, X_{2}\right) \preceq_{(2,2)-u o}^{\star}\left(Y_{1}, Y_{2}\right)$ if and only if $\widetilde{\rho}_{u_{1}, u_{2}}^{\mathrm{Sp}}\left(X_{1}, X_{2}\right) \leq \widetilde{\rho}_{u_{1}, u_{2}}^{\mathrm{Sp}}\left(Y_{1}, Y_{2}\right)$ for all $\left(u_{1}, u_{2}\right) \in[0,1]^{2}$.

\section{Tests of s-concordance orderings}

As stated in the Introduction, this paper's second aim is to provide a nonparametric statistical methodology to formally assess the s-concordance ordering of two bivariate populations. Even in the case of the usual concordance ordering, i.e. when $\mathbf{s}=(1,1)$, no procedure has been developed yet. A paper by Cebriàn, Denuit and Scaillet (2004) entitled "Testing for concordance ordering" is seemingly achieving this, but in fact the goal of these authors is to compare one bivariate population's joint distribution with a pre-specified parametric model.

\subsection{Null and alternative hypotheses}

For a fixed $\mathbf{s} \in \mathbb{N}^{2}$, the goal is to test for the stochastic dominance of a random pair $\left(Y_{1}, Y_{2}\right)$ over $\left(X_{1}, X_{2}\right)$ with respect to the lower orthant s-concordance ordering. In other words, one wants to test for

$$
\mathbb{H}_{0}^{(\mathbf{s})}:\left(X_{1}, X_{2}\right) \preceq_{\mathbf{s}-\ell_{o}}^{\star}\left(Y_{1}, Y_{2}\right) \text { against } \mathbb{H}_{1}^{(s)}:\left(X_{1}, X_{2}\right) \npreceq_{\mathbf{s}-\ell_{o}}^{\star}\left(Y_{1}, Y_{2}\right) .
$$

In view of Property 2.1, the methodology that will be developed in the sequel can easily be adapted to test for the upper orthant $\mathbf{s}$-concordance ordering $\preceq_{\mathbf{s}-u o}^{\star}$ by considering the lower orthant dominance of $\left(-Y_{1},-Y_{2}\right)$ over $\left(-X_{1},-X_{2}\right)$. 
Now a reformulation of the null and alternative hypotheses in (9) will prove useful. To this end, let $C$ and $D$ be the copulas of $\left(X_{1}, X_{2}\right)$ and $\left(Y_{1}, Y_{2}\right)$, respectively. In view of Equation (6) in Proposition 2.2, the null hypothesis $\mathbb{H}_{0}^{(\mathbf{s})}$ is true if $\mathcal{J}_{\mathbf{i}}\left(u_{1}^{\mathbb{I}\left(i_{1}=s_{1}\right)}, u_{2}^{\mathbb{I}\left(i_{2}=s_{2}\right)} ; C-D\right) \leq 0$ for all $\mathbf{i} \in \mathcal{E}_{\mathbf{s}}$. On the other side, $\mathcal{J}_{\mathbf{i}}\left(u_{1}^{\mathbb{I}\left(i_{1}=s_{1}\right)}, u_{2}^{\mathbb{I}\left(i_{2}=s_{2}\right)} ; C-D\right)>0$ for some $\mathbf{i} \in \mathcal{E}_{\mathbf{s}}$ and $\left(u_{1}, u_{2}\right) \in[0,1]^{2}$ under $\mathbb{H}_{1}^{(\mathbf{s})}$. This suggests basing a measure of s-concordance on some functional of $\mathcal{J}_{\mathbf{i}}(\cdot, \cdot ; C-D)$. Letting $\ell^{\infty}\left([0,1]^{2}\right)$ be the space of bounded functions on $[0,1]^{2}$ equipped with the supremum norm, consider $\mu_{\kappa}: \ell^{\infty}\left([0,1]^{2}\right) \rightarrow \mathbb{R}$ for some $\kappa \geq 1$ such that for $x_{+}=\max (x, 0)$,

$$
\mu_{\kappa}(g)=\left\{\int_{0}^{1} \int_{0}^{1}\left(g\left(u_{1}, u_{2}\right)\right)_{+}^{\kappa} \mathrm{d} u_{1} \mathrm{~d} u_{2}\right\}^{1 / \kappa} .
$$

Measuring the lower orthant s-concordance of a pair $\left(X_{1}, X_{2}\right)$ with respect to $\left(Y_{1}, Y_{2}\right)$ can then be based on

$$
\Theta_{\kappa,(C, D)}^{(\mathbf{s})}=\max _{\mathbf{i} \in \mathcal{E}_{\mathbf{s}}} \mu_{\kappa}\left\{\mathcal{J}_{\mathbf{i}}\left(u_{1}^{\mathbb{I}\left(i_{1}=s_{1}\right)}, u_{2}^{\mathbb{I}\left(i_{2}=s_{2}\right)} ; C-D\right)\right\} .
$$

Combination rules others than taking the maximum over $\mathbf{i} \in \mathcal{E}_{\mathbf{s}}$ could be considered as well, e.g. the sum. However, the most interesting situations are those when $\mathbf{s} \leq(2,2)$, in which case $\mathcal{E}_{\mathbf{s}}=\{\mathbf{s}\}$ has only one element. Because $\mu_{\kappa}$ is such that $\mu_{\kappa}(g) \geq 0$, with equality if and only if $g\left(u_{1}, u_{2}\right) \leq 0$ a.s. for $\left(u_{1}, u_{2}\right) \in[0,1]^{2}$, $\mathbb{H}_{0}^{(\mathbf{s})}$ holds if and only if $\Theta_{\kappa,(C, D)}^{(\mathbf{s})}=0$. The null and alternative hypotheses stated in (9) may therefore be reformulated alternatively as

$$
\mathbb{H}_{0}^{(\mathbf{s})}: \Theta_{\kappa,(C, D)}^{(\mathbf{s})}=0 \text { against } \mathbb{H}_{1}^{(\mathbf{s})}: \Theta_{\kappa,(C, D)}^{(\mathbf{s})}>0
$$

\subsection{Test statistics and asymptotics under $\alpha$-mixing}

This subsection provides an empirical version of $\Theta_{\kappa,(C, D)}^{(\mathbf{s})}$ and investigates its asymptotic behavior under a setup of serially dependent observations. Specifically, it will be assumed that the data at hand are realizations of strongly stationary processes that satisfy the $\alpha$-mixing assumption. This notion is very general, as it is shared by many popular time series models like autoregressive and GARCH processes. Specifically, following, e.g., Bradley (2005), Carrasco and Chen (2002) or Rio (2000), consider a process $\left(Z_{t}\right)_{t \in \mathbb{Z}}$ and define

$$
\alpha(\ell)=\sup _{t \in \mathbb{Z}}\left\{\sup _{(A, B) \in \mathcal{F}_{t} \times \mathcal{F}_{t+\ell}^{\star}}|\mathbb{P}(A \cap B)-\mathbb{P}(A) \mathbb{P}(B)|\right\},
$$

where $\mathcal{F}_{t}$ and $\mathcal{F}_{t}^{\star}$ are the $\sigma$-fields $\mathcal{F}_{t}=\sigma\left\{Z_{i}, i \leq t\right\}$ and $\mathcal{F}_{t}^{\star}=\sigma\left\{Z_{i}, i>t\right\}$, respectively. Then $\left(Z_{t}\right)_{t \in \mathbb{Z}}$ is said to be $\alpha$-mixing if $\alpha(\ell) \rightarrow 0$ as $\ell \rightarrow \infty$.

Now, let $\left(X_{11}, X_{12}\right), \ldots,\left(X_{n 1}, X_{n 2}\right)$ be a realization of a strongly stationary process $\left(X_{t 1}, X_{t 2}\right)_{t \in \mathbb{Z}}$ that is $\alpha$-mixing; also assume that for all $t \in \mathbb{Z}$, the 
marginal distributions of $\left(X_{t 1}, X_{t 2}\right)$ are continuous and $C$ is its unique copula. Consider another sample $\left(Y_{11}, Y_{12}\right), \ldots,\left(Y_{m 1}, Y_{m 2}\right)$, independent of the first one, that is a realization of a strongly stationary $\alpha$-mixing process $\left(Y_{t 1}, Y_{t 2}\right)_{t \in \mathbb{Z}}$ with copula $D$. Under these conditions, nonparametric estimators of $C$ and $D$ are provided by the empirical copulas, namely

$$
\begin{aligned}
C_{n}\left(u_{1}, u_{2}\right) & =\frac{1}{n} \sum_{i=1}^{n} \mathbb{I}\left(\widehat{U}_{i 1} \leq u_{1}, \widehat{U}_{i 2} \leq u_{2}\right) \\
\text { and } D_{m}\left(u_{1}, u_{2}\right) & =\frac{1}{m} \sum_{i=1}^{m} \mathbb{I}\left(\widehat{V}_{i 1} \leq u_{1}, \widehat{V}_{i 2} \leq u_{2}\right),
\end{aligned}
$$

where $n \widehat{U}_{i 1}$ (resp. $m \widehat{V}_{i 1}$ ) is the rank of $X_{i 1}$ (resp. $Y_{i 1}$ ) among $X_{11}, \ldots, X_{n 1}$ (resp. $\left.Y_{11}, \ldots, Y_{m 1}\right)$, and similarly for $n \widehat{U}_{i 2}$ (resp. $m \widehat{V}_{i 2}$ ). An empirical plug-in version of $\Theta_{\kappa,(C, D)}^{(\mathbf{s})}$ defined in (11) is then

$$
\Theta_{\kappa,(C, D)}^{(\mathbf{s})}=\max _{\mathbf{i} \in \mathcal{E}_{\mathbf{s}}} \mu_{\kappa}\left\{\mathcal{J}_{\mathbf{i}}\left(u_{1}^{\mathbb{I}\left(i_{1}=s_{1}\right)}, u_{2}^{\mathbb{I}\left(i_{2}=s_{2}\right)} ; C_{n}-D_{m}\right)\right\} .
$$

As a first step, the following proposition establishes the asymptotic behavior of $\mathcal{J}_{\mathbf{i}}\left(\cdot, \cdot ; C_{n}-D_{m}\right)$ for any $\mathbf{i} \in \mathbb{N}^{2}$. This result can be seen somewhat as a copula version of Lemma 1 of Barrett and Donald (2003) about the iterated sample and population cdf's in the univariate case, i.e. $\sqrt{n}\left\{\mathcal{I}_{s}\left(\cdot ; F_{n}\right)-\mathcal{I}_{s}(\cdot ; F)\right\}$, where $\left.\mathcal{I}_{1}(u ; F)\right\}=F(u)$ and $\left.\left.\mathcal{I}_{s}(u ; F)\right\}=\int_{0}^{u} \mathcal{I}_{s-1}(x ; F)\right\} \mathrm{d} x, s>1$. Before stating the result, the concept of a regular copula is reminded.

Definition 4.1 (Regular copula). A bivariate copula $C$ is said to be regular if its first-order partial derivatives $\dot{C}_{1}\left(u_{1}, u_{2}\right)=\partial C\left(u_{1}, u_{2}\right) / \partial u_{1}$ and $\dot{C}_{2}\left(u_{1}, u_{2}\right)=$ $\partial C\left(u_{1}, u_{2}\right) / \partial u_{2}$ exist on $[0,1]^{2}$ and are continuous, respectively, on the sets $\left\{\left(u_{1}, u_{2}\right): 0<u_{1}<1\right\}$ and $\left\{\left(u_{1}, u_{2}\right): 0<u_{2}<1\right\}$.

Now let $\mathbb{B}_{C}$ be a Gaussian process on $[0,1]^{2}$ with mean zero such that for $\left(U_{\ell 1}, U_{\ell 2}\right)=\left(F_{1}\left(X_{\ell 1}\right), F_{2}\left(X_{\ell 2}\right)\right)$, the covariance function of $\mathbb{B}_{C}$ is given by

$$
\Gamma_{\mathbb{B}_{C}}\left(u_{1}, u_{2}, u_{1}^{\prime}, u_{2}^{\prime}\right)=\sum_{\ell \in \mathbb{Z}} \operatorname{cov}\left\{\mathbb{I}\left(U_{01} \leq u_{1}, U_{02} \leq u_{2}\right), \mathbb{I}\left(U_{\ell 1} \leq u_{1}^{\prime}, U_{\ell 2} \leq u_{2}^{\prime}\right)\right\} .
$$

Define a similar process $\mathbb{B}_{D}$ based on $\left(V_{\ell 1}, V_{\ell 2}\right)=\left(G_{1}\left(Y_{\ell 1}\right), G_{2}\left(Y_{\ell 2}\right)\right)$.

Proposition 4.1. Assume that the respective $\alpha$-mixing coefficients of strictly stationary processes $\left(X_{t 1}, X_{t 2}\right)_{t \in \mathbb{Z}}$ and $\left(Y_{t 1}, Y_{t 2}\right)_{t \in \mathbb{Z}}$ are such that $\alpha(\ell)=$ $O\left(\ell^{-6-\varepsilon}\right)$ for some $\varepsilon \in(0,1 / 2]$. Then, if the copula $C$ of $\left(X_{t 1}, X_{t 2}\right)$ and the copula $D$ of $\left(Y_{t 1}, Y_{t 2}\right)$ are regular,

$$
\mathbb{L}_{n, m}^{(\mathbf{i})}=\sqrt{\frac{n m}{n+m}}\left\{\mathcal{J}_{\mathbf{i}}\left(\cdot, \cdot ; C_{n}-D_{m}\right)-\mathcal{J}_{\mathbf{i}}(\cdot, \cdot ; C-D)\right\}
$$

converges weakly in the space $\ell^{\infty}\left([0,1]^{2}\right)$ to $\mathbb{L}^{(\mathbf{i})}=\mathcal{J}_{\mathbf{i}}(\cdot, \cdot ; \sqrt{1-\omega} \mathbb{C}-\sqrt{\omega} \mathbb{D})$, where $\lim _{n, m \rightarrow \infty} n /(n+m)=\omega \in(0,1)$, and

$$
\mathbb{C}\left(u_{1}, u_{2}\right)=\mathbb{B}_{C}\left(u_{1}, u_{2}\right)-\dot{C}_{1}\left(u_{1}, u_{2}\right) \mathbb{B}_{C}\left(u_{1}, 1\right)-\dot{C}_{2}\left(u_{1}, u_{2}\right) \mathbb{B}_{C}\left(1, u_{2}\right),
$$




$$
\text { and } \mathbb{D}\left(u_{1}, u_{2}\right)=\mathbb{B}_{D}\left(u_{1}, u_{2}\right)-\dot{D}_{1}\left(u_{1}, u_{2}\right) \mathbb{B}_{D}\left(u_{1}, 1\right)-\dot{D}_{2}\left(u_{1}, u_{2}\right) \mathbb{B}_{D}\left(1, u_{2}\right) \text {. }
$$

This weak convergence holds jointly for all $\mathbf{i} \in \mathbb{N}^{2}$.

\subsection{Decision rule, significance level and consistency}

Based on the null and alternative hypotheses of s-concordance ordering as reformulated in (12), it is suggested to reject $\mathbb{H}_{0}^{(\mathbf{s})}$ in favour of $\mathbb{H}_{1}^{(\mathbf{s})}$ for large values of $\Theta_{\kappa,(n, m)}^{(\mathbf{s})}$; here, $\kappa$ refer to some functional $\mu_{\kappa}$ as defined in (10). Observe that with $\mathbb{L}_{n, m}^{(\mathbf{i})}$ defined in (14), and since $\mu_{\kappa}(r g)=r \mu_{\kappa}(g)$ for $r \in \mathbb{R}^{+}$,

$$
\begin{aligned}
\sqrt{\frac{n m}{n+m}} \Theta_{\kappa,(n, m)}^{(\mathbf{s})} & =\max _{\mathbf{i} \in \mathcal{E}_{\mathbf{s}}} \mu_{\kappa}\left\{\mathbb{L}_{n, m}^{(\mathbf{i})}\left(u_{1}^{\mathbb{I}\left(i_{1}=s_{1}\right)}, u_{2}^{\mathbb{I}\left(i_{2}=s_{2}\right)}\right)\right. \\
& \left.+\sqrt{\frac{n m}{n+m}} \mathcal{J}_{\mathbf{i}}\left(u_{1}^{\mathbb{I}\left(i_{1}=s_{1}\right)}, u_{2}^{\mathbb{I}\left(i_{2}=s_{2}\right)} ; C-D\right)\right\} .
\end{aligned}
$$

Since under the null hypothesis $\mathbb{H}_{0}^{(\mathbf{s})}, \mathcal{J}_{\mathbf{i}}\left(u_{1}^{\mathbb{I}\left(i_{1}=s_{1}\right)}, u_{2}^{\mathbb{I}\left(i_{2}=s_{2}\right)} ; C-D\right) \leq 0$ almost surely for all $\mathbf{i} \in \mathcal{E}_{\mathbf{s}}$, and because $\mu_{\kappa}(g+\Delta) \leq \mu_{\kappa}(g)$ when $\Delta \leq 0$ a.s.,

$$
\sqrt{\frac{n m}{n+m}} \Theta_{\kappa,(n, m)}^{(\mathbf{s})} \leq \max _{\mathbf{i} \in \mathcal{E}_{\mathbf{s}}} \mu_{\kappa}\left(\mathbb{L}_{n, m}^{(\mathbf{i})}\left(u_{1}^{\mathbb{I}\left(i_{1}=s_{1}\right)}, u_{2}^{\mathbb{I}\left(i_{2}=s_{2}\right)}\right)\right) .
$$

It follows that under $\mathbb{H}_{0}^{(\mathbf{s})}$ (and as long as the conditions of Proposition 4.1 hold),

$$
\begin{aligned}
& \lim _{n, m \rightarrow \infty} \mathbb{P}\left(\sqrt{\frac{n m}{n+m}} \Theta_{\kappa,(n, m)}^{(\mathbf{s})}>x\right) \\
& \leq \lim _{n, m \rightarrow \infty} \mathbb{P}\left\{\max _{\mathbf{i} \in \mathcal{E}_{\mathbf{s}}} \mu_{\kappa}\left(\mathbb{L}_{n, m}^{(\mathbf{i})}\left(u_{1}^{\mathbb{I}\left(i_{1}=s_{1}\right)}, u_{2}^{\mathbb{I}\left(i_{2}=s_{2}\right)}\right)\right)>x\right\} \\
& =\bar{\beta}_{\kappa}^{(\mathbf{s})}(x),
\end{aligned}
$$

where $\bar{\beta}_{\kappa}^{(\mathbf{s})}$ is the survival function of $\max _{\mathbf{i} \in \mathcal{E}_{\mathbf{s}}} \mu_{\kappa}\left(\mathbb{L}^{(\mathbf{i})}\left(u_{1}^{\mathbb{I}\left(i_{1}=s_{1}\right)}, u_{2}^{\mathbb{I}\left(i_{2}=s_{2}\right)}\right)\right)$. Therefore, the test whose decision rule is to reject $\mathbb{H}_{0}^{(\mathbf{s})}$ when

$$
\sqrt{\frac{n m}{n+m}} \Theta_{\kappa,(n, m)}^{(\mathbf{s})}>\left(\bar{\beta}_{\kappa}^{(\mathbf{s})}\right)^{-1}(\alpha)
$$

has an asymptotic type I error of at most $\alpha$. Hence, the test has a significance level equals to $\alpha$ as understood by Lehmann (1986) in the case of a composite null hypothesis. In the current context, it means that the test based on $\Theta_{\kappa,(n, m)}^{(\mathbf{s})}$ will have a rejection rate that will never exceed $\alpha$ for any pair of copulas $C, D$ such that $\Theta_{\kappa,(C, D)}^{(\mathbf{s})}=0$; the asymptotic level is exactly $\alpha$ when $C=D$.

A violation of $\mathbb{H}_{0}^{(\mathbf{s})}$ means that there is a set $\mathcal{B} \subset[0,1]^{2}$ of non-null Lebesgue measure such that for some $\mathbf{i} \in \mathcal{E}_{\mathbf{s}}, \mathcal{J}_{\mathbf{i}}\left(u_{1}^{\mathbb{I}\left(i_{1}=s_{1}\right)}, u_{2}^{\mathbb{I}\left(i_{2}=s_{2}\right)} ; C-D\right)>0$ for $\left(u_{1}, u_{2}\right) \in \mathcal{B}$. It can then be deduced from $(15)$ that under $\mathbb{H}_{1}^{(\mathbf{s})}$, 


$$
\begin{aligned}
& \lim _{n, m \rightarrow \infty} \mathbb{P}\left\{\sqrt{\frac{n m}{n+m}} \Theta_{\kappa,(n, m)}^{(s)}>\left(\bar{\beta}_{\kappa}^{(\mathbf{s})}\right)^{-1}(\alpha)\right\} \\
&=\lim _{n, m \rightarrow \infty} \mathbb{P}\left\{\operatorname { m a x } _ { \mathbf { i } \in \mathcal { E } _ { \mathbf { s } } } \mu _ { \kappa } \left(\mathbb{L}_{n, m}^{(\mathbf{i})}\left(u_{1}^{\mathbb{I}\left(i_{1}=s_{1}\right)}, u_{2}^{\mathbb{I}\left(i_{2}=s_{2}\right)}\right)\right.\right. \\
& \\
&\left.\left.\quad+\sqrt{\frac{n m}{n+m}} \mathcal{J}_{\mathbf{i}}\left(u_{1}^{\mathbb{I}\left(i_{1}=s_{1}\right)}, u_{2}^{\mathbb{I}\left(i_{2}=s_{2}\right)} ; C-D\right)\right)>\left(\bar{\beta}_{\kappa}^{(\mathbf{s})}\right)^{-1}(\alpha)\right\} \\
&= 1 .
\end{aligned}
$$

The test based on $\Theta_{\kappa,(n, m)}^{(s)}$ is therefore consistent under general alternatives.

\section{Performing the tests}

\subsection{Estimation of the critical value}

In order to estimate the asymptotic critical value $\left(\bar{\beta}_{\kappa}^{(\mathbf{s})}\right)^{-1}(\alpha)$, one needs to estimate the distribution function $\beta_{\kappa}^{(\mathbf{s})}(x)=\mathbb{P}\left\{\max _{\mathbf{i} \in \mathcal{E}_{\mathbf{s}}} \mu_{\kappa}\left(\mathbb{L}^{(\mathbf{i})}\left(u_{1}^{\mathbb{I}\left(i_{1}=s_{1}\right)}, u_{2}^{\mathbb{I}\left(i_{2}=s_{2}\right)}\right) \leq\right.\right.$ $x\}$. This is not an easy task, since the limit process $\mathbb{L}^{(\mathrm{i})}$ depends on the unknown copulas $C$ and $D$ under $\mathbb{H}_{0}^{(s)}$. The adopted strategy will be based on the multiplier bootstrap for empirical processes as described by Kosorok (2008) and adapted to empirical copulas under $\alpha$-mixing by Bücher and Ruppert (2013); the latter is a generalization to time series of the multiplier method for empirical copulas as described for instance by Rémillard and Scaillet (2009).

Definition 5.1 (Serial multipliers). A serial multiplier sample associated to sample data of size $n$ is a realization $\boldsymbol{\xi}=\left(\xi_{1}, \ldots, \xi_{n}\right)$ of a strictly stationary process $\left(\xi_{t}\right)_{t \in \mathbb{Z}}$ that is independent of the data process and such that

(i) $\xi_{t}$ is independent of $\xi_{t+h}$ for all $|h| \geq r \ell_{n}$, where $r \in \mathbb{R}$ is a constant and as $n \rightarrow \infty, \ell_{n} \rightarrow \infty$ and $\ell_{n} / n \rightarrow 0$;

(ii) all central moments of $\xi_{t}$ are bounded, $\mathrm{E}\left(\xi_{t}\right)=1$ and $\operatorname{cov}\left(\xi_{t}, \xi_{t+h}\right)=$ $\phi\left(h / \ell_{n}\right)$, where $\phi$ is bounded and symmetric around zero.

Consider two independent serial multiplier samples $\boldsymbol{\xi}=\left(\xi_{1}, \ldots, \xi_{n}\right)$ and $\gamma=\left(\gamma_{1}, \ldots, \gamma_{m}\right)$ that are associated, respectively, to the observed data, i.e. the independent samples $\left(X_{11}, X_{12}\right), \ldots,\left(X_{n 1}, X_{n 2}\right)$ and $\left(Y_{11}, Y_{12}\right), \ldots,\left(Y_{m 1}, Y_{m 2}\right)$. Then, for $\omega_{n, m}=n /(n+m)$, the multiplier version of $\mathbb{L}^{(\mathbf{i})}$ is defined by

$$
\widehat{\mathbb{L}}_{n, m}^{(\mathbf{i})}=\mathcal{J}_{\mathbf{i}}\left(\cdot, \cdot ; \sqrt{1-\omega_{n, m}} \widehat{\mathbb{C}}_{n}-\sqrt{\omega_{n, m}} \widehat{\mathbb{D}}_{m}\right),
$$

where $\widehat{\mathbb{C}}_{n}$ and $\widehat{\mathbb{D}}_{m}$ are the multiplier versions of the empirical processes $\mathbb{C}_{n}=$ $\sqrt{n}\left(C_{n}-C\right)$ and $\mathbb{D}_{m}=\sqrt{m}\left(D_{m}-D\right)$, namely

$$
\begin{aligned}
\widehat{\mathbb{C}}_{n}\left(u_{1}, u_{2}\right)= & \frac{1}{n} \sum_{i=1}^{n} \Xi_{i}\left\{\mathbb{I}\left(\widehat{U}_{i 1} \leq u_{1}, \widehat{U}_{i 2} \leq u_{2}\right)\right. \\
& -\dot{C}_{n 1}\left(u_{1}, u_{2}\right) \mathbb{I}\left(\widehat{U}_{i 1} \leq u_{1}\right)-\dot{C}_{n 2}\left(u_{1}, u_{2}\right) \mathbb{I}\left(\widehat{U}_{i 2} \leq u_{2}\right\}
\end{aligned}
$$




$$
\text { and } \begin{aligned}
\widehat{\mathbb{D}}_{m}\left(u_{1}, u_{2}\right)= & \frac{1}{m} \sum_{i=1}^{m} \Gamma_{i}\left\{\mathbb{I}\left(\widehat{V}_{i 1} \leq u_{1}, \widehat{V}_{i 2} \leq u_{2}\right)\right. \\
& -\dot{D}_{m 1}\left(u_{1}, u_{2}\right) \mathbb{I}\left(\widehat{V}_{i 1} \leq u_{1}\right)-\dot{D}_{m 2}\left(u_{1}, u_{2}\right) \mathbb{I}\left(\widehat{V}_{i 2} \leq u_{2}\right\}
\end{aligned}
$$

where for $\bar{\xi}=\left(\xi_{1}+\cdots+\xi_{n}\right) / n$ and $\bar{\gamma}=\left(\gamma_{1}+\cdots+\gamma_{m}\right) / m, \Xi_{i}=\left(\xi_{i} / \bar{\xi}\right)-1$ and $\Gamma_{i}=\left(\gamma_{i} / \bar{\gamma}\right)-1$. In the above expressions, $\dot{C}_{n 1}, \dot{C}_{n 2}$ (resp. $\left.\dot{D}_{m 1}, \dot{D}_{m 2}\right)$ are uniformly consistent estimators of $\dot{C}_{1}, \dot{C}_{2}$ (resp. $\dot{D}_{1}, \dot{D}_{2}$ ). It is also assumed that for any $\epsilon \in(0,1 / 2)$,

$$
\sup _{\substack{u_{1} \in[\epsilon, 1-\epsilon] \\ u_{2} \in[0,1]}}\left|\dot{C}_{n 1}\left(u_{1}, u_{2}\right)-\dot{C}_{1}\left(u_{1}, u_{2}\right)\right| \text { and } \sup _{\substack{u_{1} \in[0,1], u_{2} \in[\epsilon, 1-\epsilon]}}\left|\dot{C}_{n 2}\left(u_{1}, u_{2}\right)-\dot{C}_{2}\left(u_{1}, u_{2}\right)\right|
$$

converge in probability to zero, and similarly for $\dot{D}_{m 1}, \dot{D}_{m 2}$. An estimator that possess these properties is described in subsection 6.1 .

Proposition 5.1. Assume that the respective $\alpha$-mixing coefficients of strictly stationary processes $\left(X_{t 1}, X_{t 2}\right)_{t \in \mathbb{Z}}$ and $\left(Y_{t 1}, X_{Y 2}\right)_{t \in \mathbb{Z}}$ are such that there exists $\varepsilon \in(0,1 / 2)$ with $\alpha(\ell)=O\left(\ell^{-6-\varepsilon}\right)$ and $\ell_{n}=O\left(n^{1 / 2-\varepsilon}\right)$, and for $\zeta(\varepsilon)=$ $\max \{28,\lfloor 2 / \varepsilon\rfloor+1\}, \sum_{\ell=1}^{\infty}(\ell+1)^{\zeta(\varepsilon)} \sqrt{\alpha(\ell)}<\infty$. If $C$ and $D$ are regular copulas, then for any $\mathbf{i} \in \mathbb{N}^{2},\left(\mathbb{L}_{n, m}^{(\mathbf{i})}, \widehat{\mathbb{L}}_{n, m}^{(\mathbf{i})}\right)$ converges weakly to $\left(\mathbb{L}^{(\mathbf{i})}, \widetilde{\mathbb{L}}^{(\mathbf{i})}\right)$, where $\widetilde{\mathbb{L}}^{(\mathbf{i})}$ is an independent copy of $\mathbb{L}^{(\mathbf{i})}=\mathcal{J}_{\mathbf{i}}(\cdot, \cdot ; \sqrt{1-\omega} \mathbb{C}-\sqrt{\omega} \mathbb{D})$. This weak convergence holds jointly for all $\mathbf{i} \in \mathbb{N}^{2}$.

In order to approximate the distribution $\beta_{\kappa}^{(\mathbf{s})}$ of $\max _{\mathbf{i} \in \mathcal{E}_{\mathbf{s}}} \mu_{\kappa}\left(\mathbb{L}^{(\mathbf{i})}\left(u_{1}^{\mathbb{I}\left(i_{1}=s_{1}\right)}\right.\right.$, $\left.u_{2}^{\mathbb{I}\left(i_{2}=s_{2}\right)}\right)$, the multiplier bootstrap is repeated with $B$ independent serial multiplier samples, which yields the independent replicates $\left(\widehat{\mathbb{L}}_{n, m}^{(\mathbf{i})}\right)^{(1)}, \ldots,\left(\widehat{\mathbb{L}}_{n, m}^{(\mathbf{i})}\right)^{(B)}$ of $\mathbb{L}_{n, m}^{(\mathbf{i})}$. Next letting, for each $b \in\{1, \ldots, B\}$,

$$
\left(\widehat{\Theta}_{\kappa,(n, m)}^{(\mathbf{s})}\right)^{(b)}=\max _{\mathbf{i} \in \mathcal{E}_{\mathbf{s}}} \mu_{\kappa}\left\{\left(\widehat{\mathbb{L}}_{n, m}^{(\mathbf{i})}\left(u_{1}^{\mathbb{I I}\left(i_{1}=s_{1}\right)}, u_{2}^{\mathbb{I}\left(i_{2}=s_{2}\right)}\right)\right)^{(b)}\right\}
$$

provides with $B$ independent replicates of $\Theta_{\kappa,(n, m)}^{(\mathbf{s})}$. The critical value $\left(\bar{\beta}_{\kappa}^{(\mathbf{s})}\right)^{-1}(\alpha)$ is finally estimated by the $(1-\alpha)$-th empirical percentile of these multiplier bootstrap replicates. The consistency of this estimator is a straightforward consequence of Proposition 5.1.

\subsection{Approximation of the test statistics}

From the recursive definition of $\mathcal{J}_{\mathbf{i}}$ in Equation (5), one can easily establish by induction that when $g\left(u_{1}, u_{2}\right)=\mathbb{I}\left(a_{1} \leq u_{1}, a_{2} \leq u_{2}\right)$,

$$
\mathcal{J}_{\mathbf{i}}\left(u_{1}, u_{2} ; g\right)=\frac{\left(u_{1}-a_{1}\right)_{+}^{i_{1}-1}}{\left(i_{1}-1\right) !} \times \frac{\left(u_{2}-a_{2}\right)_{+}^{i_{2}-1}}{\left(i_{2}-1\right) !} .
$$


It follows that

$$
\mathcal{J}_{\mathbf{i}}\left(u_{1}, u_{2} ; C_{n}\right)=\frac{1}{n} \sum_{i=1}^{n} \frac{\left(u_{1}-\widehat{U}_{i 1}\right)_{+}^{i_{1}-1}}{\left(i_{1}-1\right) !} \times \frac{\left(u_{2}-\widehat{U}_{i 2}\right)_{+}^{i_{2}-1}}{\left(i_{2}-1\right) !},
$$

and similarly for $\mathcal{J}_{\mathbf{i}}\left(u_{1}, u_{2} ; C_{m}\right)$. Now the test statistic $\Theta_{\kappa,(n, m)}^{(\mathbf{s})}$ defined in (13), which involves the computation of $\mu_{\kappa}\left\{\mathcal{J}_{\mathbf{i}}\left(\cdot, \cdot ; C_{n}-D_{m}\right)\right.$ for each $\mathbf{i} \in \mathcal{E}_{\mathbf{s}}$, will be based on an approximation of $\mathcal{J}_{\mathbf{i}}\left(\cdot, \cdot ; C_{n}-D_{m}\right)=\mathcal{J}_{\mathbf{i}}\left(\cdot, \cdot ; C_{n}\right)-\mathcal{J}_{\mathbf{i}}\left(\cdot, \cdot ; D_{m}\right)$ on a $\mathcal{K} \times \mathcal{K}$ grid of $[0,1]^{2}$ for $\mathcal{K} \in \mathbb{N}$ taken large enough to ensure a satisfactory numerical accuracy. Specifically, consider the product intervals

$$
\mathcal{A}_{k_{1}, k_{2}}=\left(\frac{k_{1}}{\mathcal{K}}, \frac{k_{1}+1}{\mathcal{K}}\right] \times\left(\frac{k_{2}}{\mathcal{K}}, \frac{k_{2}+1}{\mathcal{K}}\right], \quad k_{1}, k_{2} \in\{1, \ldots, \mathcal{K}\},
$$

and let $u_{k}=(k-1 / 2) / \mathcal{K}$ for $k \in\{1, \ldots, \mathcal{K}\}$. Then, define $Z^{(\mathbf{i})}, W^{(\mathbf{i})} \in \mathbb{R}^{\mathcal{K} \times \mathcal{K}}$ such that $Z_{k_{1}, k_{2}}^{(\mathbf{i})}=\mathcal{J}_{\mathbf{i}}\left(u_{k_{1}}, u_{k_{2}} ; C_{n}\right)$ and $W_{k_{1}, k_{2}}^{(\mathbf{i})}=\mathcal{J}_{\mathbf{i}}\left(u_{k_{1}}, u_{k_{2}} ; D_{m}\right)$, so that

$$
\begin{aligned}
\mathcal{J}_{\mathbf{i}}\left(u_{1}, u_{2} ; C_{n}\right) & \approx \sum_{k_{1}, k_{2}=1}^{\mathcal{K}} Z_{k_{1}, k_{2}}^{(\mathbf{i})} \mathbb{I}\left\{\left(u_{1}, u_{2}\right) \in \mathcal{A}_{k_{1}, k_{2}}\right\} \\
\text { and } \mathcal{J}_{\mathbf{i}}\left(u_{1}, u_{2} ; D_{m}\right) & \approx \sum_{k_{1}, k_{2}=1}^{\mathcal{K}} W_{k_{1}, k_{2}}^{(\mathbf{i})} \mathbb{I}\left\{\left(u_{1}, u_{2}\right) \in \mathcal{A}_{k_{1}, k_{2}}\right\} .
\end{aligned}
$$

From the definition of $\mu_{\kappa}$ in (10), an easily computable approximation is

$$
\Theta_{\kappa,(n, m)}^{(\mathbf{s})} \approx \max _{\mathbf{i} \in \mathcal{E}_{\mathbf{s}}}\left\{\frac{1}{\mathcal{K}^{2}} \sum_{k_{1}, k_{2}=1}^{\mathcal{K}}\left(Z_{k 1, k 2}^{(\mathbf{i})}-W_{k 1, k 2}^{(\mathbf{i})}\right)_{+}^{\kappa}\right\}^{1 / \kappa} .
$$

\subsection{User-friendly formulas for the multiplier versions}

First define the column vector $\mathbf{I}_{k_{1}, k_{2}}^{\mathbb{C}}=\left(I_{1, k_{1}, k_{2}}^{\mathbb{C}}, \ldots, I_{n, k_{1}, k_{2}}^{\mathbb{C}}\right)^{\top}$ such that for each $\left(k_{1}, k_{2}\right) \in\{1, \ldots, \mathcal{K}\}^{2}$ and $i \in\{1, \ldots, n\}$,

$$
\begin{aligned}
I_{i, k_{1}, k_{2}}^{\mathbb{C}}= & \mathbb{I}\left(\widehat{U}_{i 1} \leq u_{k_{1}}, \widehat{U}_{i 2} \leq u_{k_{2}}\right)-\dot{C}_{n 1}\left(u_{k_{1}}, u_{k_{2}}\right) \mathbb{I}\left(\widehat{U}_{i 1} \leq u_{k_{1}}\right) \\
& -\dot{C}_{n 2}\left(u_{k_{1}}, u_{k_{2}}\right) \mathbb{I}\left(\widehat{U}_{i 2} \leq u_{k_{2}}\right) .
\end{aligned}
$$

One can then write $\widehat{\mathbb{C}}_{n}$ compactly as $\widehat{\mathbb{C}}_{n}\left(u_{k_{1}}, u_{k_{2}}\right)=\Xi \mathbf{I}_{k_{1}, k_{2}}^{\mathbb{C}} / \sqrt{n}$, with $\Xi=$ $\left(\Xi_{1}, \ldots, \Xi_{n}\right)$. Then, letting $\widehat{Z}_{k_{1}, k_{2}}^{(\mathbf{i})}=\mathcal{J}_{\mathbf{i}}\left(u_{k_{1}}, u_{k_{2}} ; \widehat{\mathbb{C}}_{n}\right)$, one has $\widehat{Z}_{k_{1}, k_{2}}^{(1,1)}=$ $\widehat{\mathbb{C}}_{n}\left(u_{k_{1}}, u_{k_{2}}\right)$, and in view of (5), one obtains recursively

$$
\begin{aligned}
\widehat{Z}_{k_{1}, k_{2}}^{(2,1)} & =\int_{0}^{u_{k_{1}}} \widehat{\mathbb{C}}_{n}\left(x_{1}, u_{k_{2}}\right) \mathrm{d} x_{1} \approx \frac{1}{\mathcal{K}} \sum_{\ell=1}^{\mathcal{K}} \mathbb{I}\left(u_{\ell} \leq u_{k_{1}}\right) \widehat{Z}_{\ell, k_{2}}^{(1,1)} \\
\text { and } \widehat{Z}_{k_{1}, k_{2}}^{(1,2)} & =\int_{0}^{u_{k_{2}}} \widehat{\mathbb{C}}_{n}\left(u_{k_{1}}, x_{2}\right) \mathrm{d} x_{2} \approx \frac{1}{\mathcal{K}} \sum_{\ell=1}^{\mathcal{K}} \mathbb{I}\left(u_{\ell} \leq u_{k_{2}}\right) \widehat{Z}_{k_{1}, \ell}^{(1,1)},
\end{aligned}
$$


and so on. For instance, one obtains

$$
\widehat{Z}_{k_{1}, k_{2}}^{(2,2)} \approx \frac{1}{\mathcal{K}^{2}} \sum_{\ell_{1}, \ell_{2}=1}^{\mathcal{K}} \mathbb{I}\left(u_{\ell_{1}} \leq u_{k_{1}}, u_{\ell 2} \leq u_{k_{2}}\right) \widehat{Z}_{\ell_{1}, \ell_{2}}^{(1,1)}
$$

One proceeds similarly for $\widehat{W}_{k_{1}, k_{2}}^{(\mathbf{i})}=\mathcal{J}_{\mathbf{i}}\left(u_{k_{1}}, u_{k_{2}} ; \widehat{\mathbb{D}}_{m}\right)$ by letting

$$
\begin{aligned}
I_{i, k_{1}, k_{2}}^{\mathbb{D}}= & \mathbb{I}\left(\widehat{V}_{i 1} \leq u_{k_{1}}, \widehat{V}_{i 2} \leq u_{k_{2}}\right)-\dot{D}_{m 1}\left(u_{k_{1}}, u_{k_{2}}\right) \mathbb{I}\left(\widehat{V}_{i 1} \leq u_{k_{1}}\right) \\
& -\dot{D}_{m 2}\left(u_{k_{1}}, u_{k_{2}}\right) \mathbb{I}\left(\widehat{V}_{i 2} \leq u_{k_{2}}\right)
\end{aligned}
$$

and for a set of (rescaled) multipliers $\Gamma=\left(\Gamma_{1}, \ldots, \Gamma_{m}\right)$ independent of $\Xi$, $\widehat{\mathbb{D}}_{m}\left(u_{k_{1}}, u_{k_{2}}\right)=\Gamma \mathbf{I}_{k_{1}, k_{2}}^{\mathbb{D}} / \sqrt{m}$, where $\mathbf{I}_{k_{1}, k_{2}}^{\mathbb{D}}=\left(I_{1, k_{1}, k_{2}}^{\mathbb{D}}, \ldots, I_{m, k_{1}, k_{2}}^{\mathbb{D}}\right)^{\top}$. An approximation of $\widehat{\Theta}_{\kappa,(n, m)}^{(\mathbf{s})}$ is finally obtained by letting

$$
\widehat{\Theta}_{\kappa,(n, m)}^{(\mathbf{s})} \approx \max _{\mathbf{i} \in \mathcal{E}_{\mathbf{s}}}\left\{\frac{1}{\mathcal{K}^{2}} \sum_{k_{1}, k_{2}=1}^{\mathcal{K}}\left(\sqrt{1-\omega_{n, m}} \widehat{Z}_{k_{1}, k_{2}}^{(\mathbf{i})}-\sqrt{\omega_{n, m}} \widehat{W}_{k_{1}, k_{2}}^{(\mathbf{i})}\right)_{+}^{\kappa}\right\}^{1 / \kappa} .
$$

\section{Sampling properties of the tests of s-concordance orderings}

\subsection{Preliminaries}

The ability of the tests of $(1,1)$-concordance and $(2,2)$-concordance orderings to keep their nominal $5 \%$ level, as well as their power against selected alternatives, is studied in this section. The test statistics that will be investigated are those based on the functional $\mu_{\kappa}$ defined in (10) when $\kappa \in\{1,2, \infty\}$. While $\mu_{1}$ and $\mu_{2}$ are global distance measures of the Cramér-von Mises type,

$$
\mu_{\infty}(g)=\sup _{\left(u_{1}, u_{2}\right) \in[0,1]^{2}}\left(g\left(u_{1}, u_{2}\right)\right)_{+}
$$

is related to the Kolmogorov-Smirnov distance. These functionals are approximated on a grid of size $\mathcal{K} \times \mathcal{K}=25 \times 25$, as described in subsections 5.2-5.3. For all the results that will be presented, the probabilities of rejection of the null hypothesis have been estimated from 1000 replicates, each based on $B=1000$ multiplier bootstrap samples. The estimation of the partial derivatives $\dot{C}_{1}, \dot{C}_{2}$, $\dot{D}_{1}$ and $\dot{D}_{2}$ are based on a finite-difference estimator. Explicitly, the estimator of $\dot{C}_{1}$ is given for $b_{n}=b / \sqrt{n}$, with $b \in \mathbb{N}$, by

$$
\dot{C}_{n 1}\left(u_{1}, u_{2}\right)= \begin{cases}\frac{C_{n}\left(2 b_{n}, u_{2}\right)}{2 b_{n}}, & \text { if } u_{1}<b_{n} ; \\ \frac{C_{n}\left(u_{1}+b_{n}, u_{2}\right)-C_{n}\left(u_{1}-b_{n}, u_{2}\right)}{2 b_{n}}, & \text { if } b_{n} \leq u_{1} \leq 1-b_{n} ; \\ \frac{C_{n}\left(1, u_{2}\right)-C_{n}\left(1-2 b_{n}, u_{2}\right)}{2 b_{n}}, & \text { if } u_{1}>1-b_{n},\end{cases}
$$

and similarly for $\dot{C}_{n 2}, \dot{D}_{m 1}$ and $\dot{D}_{m 2}$; in the sequel, $b=1$. 


\subsection{Tests of $(1,1)$-concordance ordering in the i.i.d. case}

One first considers the size and power of the tests of concordance ordering, i.e. of

$\mathbb{H}_{0}^{(1,1)}$ against $\mathbb{H}_{1}^{(1,1)}$, under the i.i.d. setup where one is willing to assume serial independence in both time series. In that case, the multiplier random variables can be taken i.i.d., i.e. $\ell_{n}=\ell_{m}=1$, and Exponential with mean 1. The results on the estimated probabilities of rejection of the null hypothesis are found in Table 3 for $(n, m) \in\{(100,100),(100,200),(200,200)\}$.

The four scenarios in the upper part of Table 3 are under the null hypothesis. In the case of the first two scenarios, i.e. when $C$ and $D$ are normal copulas, the null hypothesis holds strictly in the sense that $C=D$; in that case, the three tests are rather good at holding their $5 \%$ nominal level, except for small sample sizes $(n=m=100)$ and a high level of dependence $\left(\tau_{C}=\tau_{D}=2 / 3\right)$. The other two scenarios are cases where $\mathbb{H}_{0}^{(1,1)}$ holds, but not strictly, i.e. $C \neq D$; it is therefore not surprising that the probabilities of rejection are far below the $5 \%$ nominal level of the tests.

All the other entries in Table 3 are obtained under the alternative hypothesis. Overall, the power is an increasing function of the departure from $\mathbb{H}_{0}^{(1,1)}$ as measured by $\Theta_{\infty,(C, D)}^{(1,1)}$. However, when the value of $\Theta_{\infty,(C, D)}^{(1,1)}$ is small (say < $.02)$, the power is often below the nominal level. Otherwise, the power of the three tests is very good and increases with the sample sizes, a consequence of their asymptotic consistency. It is hard to identify a statistic that is uniformly the best, but the tests based on $\mu_{1}$ and $\mu_{2}$ are generally preferable to that using $\mu_{\infty}$. The test based on $\mu_{2}$ is generally the most powerful when $D$ is a Clayton copula, and that using $\mu_{1}$ is the best when $D$ is Plackett.

\subsection{Tests of (2,2)-concordance ordering in the i.i.d. case}

A study similar to that presented in subsection 6.2 has been conducted for testing $\mathbb{H}_{0}^{(2,2)}$ against $\mathbb{H}_{1}^{(2,2)}$. Since $\preceq_{(1,1)-\ell o}^{\star}$ implies $\preceq_{(2,2)-\ell o}^{\star}$, only the scenarios for which $\npreceq(1,1)-\ell$ o as identified in Table 1 , have been considered . The results on the estimated probabilities of rejection of the null hypothesis are found in Table 4 . First note that the four scenarios in the upper part of Table 4 concern non strict null hypotheses, i.e. situations where $C \neq D$; this explains why the probabilities of rejection are below the $5 \%$ nominal level in that case. The remaining six entries in the bottom part of Table 4 correspond to scenarios under $\mathbb{H}_{1}^{(2,2)}$. As expected, the power of the tests increases with the sample sizes, as well as according to the value of $\Theta_{\infty,(C, D)}^{(2,2)}$. Here, the test based on the functional $\mu_{\infty}$ is clearly the most powerful against all kinds of alternatives.

\subsection{Size and power under Gaussian serial dependence}

When there is temporal dependence between the observations, one has to rely on the serial multiplier method. In the sequel, one follows Bücher and Ruppert (2013) and let $\left(\zeta_{j}\right)_{j \in \mathbb{Z}}$ be a process of independent $\operatorname{Gamma}(q, q)$ ran- 
TABLE 3. Percentages of rejection, as estimated from 1000 replicates, of the tests for the $(1,1)$-concordance ordering hypothesis based on $\Theta_{1,(n, m)}^{(1,1)}$, $\Theta_{2,(n, m)}^{(1,1)}$ and $\Theta_{\infty,(n, m)}^{(1,1)}$ under the i.i.d. setup involving the Normal (N), Clayton $(C \ell)$, Gumbel (Gu) and Plackett $(P \ell)$ copulas

\begin{tabular}{|c|c|c|c|c|c|c|c|c|c|c|c|c|c|}
\hline \multirow[b]{2}{*}{$C$} & \multicolumn{4}{|c|}{ Scenario } & \multicolumn{3}{|c|}{$n=100, m=100$} & \multicolumn{3}{|c|}{$n=100, m=200$} & \multicolumn{3}{|c|}{$n=200, m=200$} \\
\hline & $\tau_{C}$ & $D$ & $\tau_{D}$ & $\Theta_{\infty,(C, D)}^{(1,1)}$ & $\Theta_{1,(n, m)}^{(1,1)}$ & $\Theta_{2,(n, m)}^{(1,1)}$ & $\Theta_{\infty,(n, m)}^{(1,1)}$ & $\Theta_{1,(n, m)}^{(1,1)}$ & $\Theta_{2,(n, m)}^{(1,1)}$ & $\Theta_{\infty,(n, m)}^{(1,1)}$ & $\Theta_{1,(n, m)}^{(1,1)}$ & $\Theta_{2,(n, m)}^{(1,1)}$ & $\Theta_{\infty,(n, m)}^{(1,1)}$ \\
\hline $\mathrm{N}$ & $1 / 3$ & $\mathrm{~N}$ & $1 / 3$ & 0 & 5.1 & 4.4 & 3.6 & 5.5 & 5.1 & 3.3 & 3.6 & 3.4 & 2.6 \\
\hline $\mathrm{N}$ & $2 / 3$ & $\mathrm{~N}$ & $2 / 3$ & 0 & 2.2 & 2.2 & 1.9 & 4.1 & 4.2 & 3.7 & 3.3 & 3.6 & 2.8 \\
\hline $\mathrm{P} \ell$ & .35 & $\mathrm{C} \ell$ & $2 / 3$ & 0 & 0.0 & 0.1 & 0.1 & 0.0 & 0.0 & 0.0 & 0.0 & 0.0 & 0.0 \\
\hline $\mathrm{Gu}$ & .15 & $\mathrm{P} \ell$ & $2 / 3$ & 0 & 0.0 & 0.0 & 0.0 & 0.0 & 0.0 & 0.0 & 0.0 & 0.0 & 0.0 \\
\hline $\mathrm{N}$ & .40 & $\mathrm{~N}$ & $1 / 3$ & .0167 & 20.8 & 18.5 & 11.8 & 25.9 & 23.0 & 15.9 & 27.4 & 25.2 & 18.8 \\
\hline $\mathrm{N}$ & .60 & $\mathrm{~N}$ & $1 / 3$ & .0666 & 95.1 & 93.2 & 79.4 & 99.3 & 98.8 & 92.9 & 100.0 & 100.0 & 97.3 \\
\hline $\mathrm{Gu}$ & .40 & $\mathrm{C} \ell$ & $1 / 3$ & .0469 & 24.6 & 28.0 & 23.6 & 31.9 & 39.7 & 39.2 & 46.2 & 57.6 & 55.1 \\
\hline $\mathrm{Gu}$ & .60 & $\mathrm{C} \ell$ & $1 / 3$ & .0861 & 95.9 & 95.8 & 90.2 & 98.8 & 99.2 & 97.9 & 100.0 & 100.0 & 99.7 \\
\hline $\mathrm{Gu}$ & .40 & $\mathrm{C} \ell$ & $2 / 3$ & .0169 & 0.0 & 0.0 & 0.3 & 0.0 & 0.0 & 0.3 & 0.0 & 0.0 & 0.4 \\
\hline $\mathrm{Gu}$ & .60 & $\mathrm{C} \ell$ & $2 / 3$ & .0362 & 2.0 & 5.5 & 9.1 & 2.7 & 8.2 & 16.8 & 4.1 & 16.2 & 29.4 \\
\hline $\mathrm{Gu}$ & .80 & $\mathrm{C} \ell$ & $2 / 3$ & & 85.0 & 89.7 & 72.5 & 97.7 & 98.9 & 96.0 & 99.6 & 99.9 & 99.1 \\
\hline $\mathrm{P} \ell$ & .40 & $\mathrm{C} \ell$ & $1 / 3$ & .0376 & 22.3 & 25.6 & 21.2 & 32.4 & 37.9 & 35.0 & 37.6 & 44.5 & 42.4 \\
\hline $\mathrm{P} \ell$ & .60 & $\mathrm{C} \ell$ & $1 / 3$ & .0847 & 94.7 & 95.5 & 90.8 & 98.2 & 98.8 & 97.6 & 99.8 & 100.0 & 100.0 \\
\hline $\mathrm{P} \ell$ & .40 & $\mathrm{C} \ell$ & $2 / 3$ & .0006 & 0.0 & 0.0 & 0.0 & 0.0 & 0.0 & 0.0 & 0.0 & 0.0 & 0.0 \\
\hline $\mathrm{P} \ell$ & .60 & $\mathrm{C} \ell$ & $2 / 3$ & .0208. & 0.9 & 2.3 & 4.7 & 1.5 & 3.4 & 7.5 & 2.0 & 4.2 & 9.2 \\
\hline $\mathrm{P} \ell$ & .80 & $\mathrm{C} \ell$ & $2 / 3$ & .0549. & 69.3 & 77.2 & 63.1 & 86.9 & 94.3 & 91.2 & 94.7 & 98.3 & 96.7 \\
\hline $\mathrm{Gu}$ & .40 & $\mathrm{P} \ell$ & $1 / 3$ & .0262 & 18.6 & 15.3 & 9.5 & 23.4 & 19.7 & 13.3 & 25.9 & 21.8 & 13.8 \\
\hline $\mathrm{Gu}$ & .60 & $\mathrm{P} \ell$ & $1 / 3$ & .0635 & 93.8 & 91.1 & 75.3 & 98.2 & 97.9 & 90.3 & 100.0 & 100.0 & 97.6 \\
\hline $\mathrm{Gu}$ & .40 & $\mathrm{P} \ell$ & $2 / 3$ & .0031 & 0.0 & 0.0 & 0.0 & 0.0 & 0.0 & 0.0 & 0.0 & 0.0 & 0.0 \\
\hline $\mathrm{Gu}$ & .60 & $\mathrm{P} \ell$ & $2 / 3$ & .0105. & 0.1 & 0.0 & 0.3 & 0.3 & 0.3 & 0.4 & 0.1 & 0.2 & 0.7 \\
\hline $\mathrm{Gu}$ & .80 & $\mathrm{P} \ell$ & $2 / 3$ & .0305 & 66.5 & 51.9 & 22.1 & 89.2 & 81.5 & 49.4 & 95.6 & 92.4 & 60.5 \\
\hline
\end{tabular}


TABLE 4. Percentages of rejection, as estimated from 1000 replicates, of the tests for the $(2,2)$-concordance ordering hypothesis based on $\Theta_{1,(n, m)}^{(2,2)}$, $\Theta_{2,(n, m)}^{(2,2)}$ and $\Theta_{\infty,(n, m)}^{(2,2)}$ under the i.i.d. setup involving the Gumbel (Gu) and Plackett (P $)$ copulas

\begin{tabular}{|c|c|c|c|c|c|c|c|c|c|c|c|c|c|}
\hline \multirow[b]{2}{*}{ C } & \multicolumn{4}{|c|}{ Scenario } & \multicolumn{3}{|c|}{$n=100, m=100$} & \multicolumn{3}{|c|}{$n=100, m=200$} & \multicolumn{3}{|c|}{$n=200, m=200$} \\
\hline & $\tau_{C}$ & $D$ & $\tau_{D}$ & $\Theta_{\infty,(n, m)}^{(2,2)}$ & $\Theta_{1,(n, m)}^{(2,2)}$ & $\Theta_{2,(n, m)}^{(2,2)}$ & $\Theta_{\infty,(n, m)}^{(2,2)}$ & $\Theta_{1,(n, m)}^{(2,2)}$ & $\Theta_{2,(n, m)}^{(2,2)}$ & $\Theta_{\infty,(n, m)}^{(2,2)}$ & $\Theta_{1,(n, m)}^{(2,2)}$ & $\Theta_{2,(n, m)}^{(2,2)}$ & $\Theta_{\infty,(n, m)}^{(2,2)}$ \\
\hline $\mathrm{Gu}$ & .33 & $\mathrm{C} \ell$ & $1 / 3$ & 0 & 0.2 & 0.6 & 3.3 & 0.2 & 0.5 & 2.2 & 0.3 & 0.6 & 4.7 \\
\hline $\mathrm{Gu}$ & .66 & $\mathrm{C} \ell$ & $2 / 3$ & 0 & 0.0 & 0.3 & 3.0 & 0.0 & 0.0 & 1.3 & 0.0 & 0.0 & 2.9 \\
\hline $\mathrm{Gu}$ & .25 & $\mathrm{P} \ell$ & $1 / 3$ & 0 & 0.4 & 0.3 & 0.5 & 0.1 & 0.1 & 0.2 & 0.1 & 0.1 & 0.2 \\
\hline $\mathrm{Gu}$ & .58 & $\mathrm{P} \ell$ & $2 / 3$ & 0 & 0.0 & 0.0 & 0.2 & 0.0 & 0.0 & 0.0 & 0.0 & 0.0 & 0.0 \\
\hline $\mathrm{Gu}$ & .60 & $\mathrm{C} \ell$ & $1 / 3$ & .0258 & 55.6 & 75.5 & 95.3 & 66.6 & 84.2 & 97.5 & 87.8 & 96.4 & 99.8 \\
\hline $\mathrm{Gu}$ & .80 & $\mathrm{C} \ell$ & $1 / 3$ & .0387 & 100.0 & 100.0 & 100.0 & 100.0 & 100.0 & 100.0 & 100.0 & 100.0 & 100.0 \\
\hline $\mathrm{Gu}$ & .80 & $\mathrm{C} \ell$ & $2 / 3$ & .0080 & 10.4 & 33.8 & 80.8 & 4.6 & 24.5 & 71.6 & 29.8 & 71.6 & 97.8 \\
\hline $\mathrm{Gu}$ & .60 & $\mathrm{P} \ell$ & $1 / 3$ & .0258 & 81.7 & 87.5 & 94.8 & 86.2 & 89.9 & 96.5 & 97.9 & 98.8 & 99.8 \\
\hline $\mathrm{Gu}$ & .80 & $\mathrm{P} \ell$ & $1 / 3$ & .0387 & 100.0 & 100.0 & 100.0 & 100.0 & 100.0 & 100.0 & 100.0 & 100.0 & 100.0 \\
\hline $\mathrm{Gu}$ & .80 & $\mathrm{P} \ell$ & $2 / 3$ & .0086 & 46.9 & 54.8 & 75.9 & 42.6 & 51.6 & 66.2 & 80.0 & 84.8 & 96.7 \\
\hline
\end{tabular}


dom variables with $q=\left(2 \ell_{n}-1\right)^{-1}$, where the bandwidth parameter is set to $\ell_{n}=\left\lfloor 1.1 n^{1 / 4}\right\rfloor$. Then, for each $j \in\{1, \ldots, n\}$, one defines $\xi_{j}$ as the mean of $\zeta_{j-\ell_{n}+1}, \ldots, \zeta_{j+\ell_{n}-1}$. One proceeds similarly for the second sample of size $m$.

The results reported in the upper part of Table 5 have been obtained for serial data generated from the lag-1 Gaussian autoregressive process

$$
\left(X_{t 1}, X_{t 2}\right)=\theta\left(X_{t-1,1}, X_{t-1,2}\right)+\sqrt{1-\theta^{2}}\left(\varepsilon_{t 1} \cdot \varepsilon_{t 2}\right),
$$

where $\theta \in(-1,1)$ and $\left(\varepsilon_{t 1}, \varepsilon_{t 2}\right)_{t \in \mathbb{Z}}$ is a process of centred independent Normal pairs with unit variances and correlation $\rho \in(-1,1)$. The middle part of Table 5 concerns the Gaussian moving-average process of order one defined by

$$
\left(X_{t 1}, X_{t 2}\right)=\frac{\theta\left(\varepsilon_{t-1,1}, \varepsilon_{t-1,2}\right)+\left(\varepsilon_{t 1}, \varepsilon_{t 2}\right)}{\sqrt{1-\theta^{2}}} .
$$

These processes are stationary and parameterized in such a way that the copula of $\left(X_{t 1}, X_{t 2}\right)$ is Normal with parameter $\rho$. The level of dependence of the generated time series is managed by the value of Kendall's tau via the well-known relationships $\rho_{C}=\sin \left(\pi \tau_{C} / 2\right)$ and $\rho_{D}=\sin \left(\pi \tau_{D} / 2\right)$. Only the results when $\tau_{D}=1 / 3$ are presented, since those when $\tau_{D}=2 / 3$ lead to similar conclusions. For comparison purposes, the results that have been already obtained under serial independence are reported here in the bottom part of Table 5.

Overall, the results are very similar to those in Table 3 in the case of i.i.d. data. In particular, if one looks at the cases when $\theta=0$, there is no price to pay in terms of size and power by wrongly assuming serial dependence. When $\tau_{C}=1 / 3$, the null hypothesis $\mathbb{H}_{0}^{(1,1)}$ holds strictly; in that case, the three tests keep their $5 \%$ nominal level well, whatever the kind and level of serial dependence. This is an indication that the serial multiplier method is good at replicating the behavior of the test statistics under the null hypothesis.

\subsection{Comparisons with a test of s-increasing convex order}

Suppose a setup of fixed marginals, i.e. of pairs $\left(X_{1}, X_{2}\right)$ and $\left(Y_{1}, Y_{2}\right)$ that belong to the same Fréchet class of bivariate distributions with margins $F_{1}, F_{2}$. In that case, according to Proposition 2.1 (ii), the s-increasing convex dominance of $\left(-Y_{1},-Y_{2}\right)$ over $\left(-X_{1},-X_{2}\right)$ entails that $\left(X_{1}, X_{2}\right) \preceq_{\mathrm{s}-\ell_{o}}^{\star}\left(Y_{1}, Y_{2}\right)$ as long as $F_{1}$ is $s_{1}$-concave and $F_{2}$ is $s_{2}$-concave. Therefore, if in practice one is willing to assume these constraints on the marginal distributions, simplified tests for $\mathbb{H}_{0}^{\mathbf{s}}$ against $\mathbb{H}_{1}^{\mathbf{s}}$ could be based on the observations themselves, and not on their ranks, as is mandatory when working at the level of copulas. This procedure would avoid the estimation of the partial derivatives of copulas due to the fact that these terms are missing in the (simpler) asymptotic expression of the limit.

Specifically, suppose that the goal is to test for $\widetilde{\mathbb{H}}_{0}^{\mathbf{s}}:\left(-X_{1},-X_{2}\right) \preceq_{\mathbf{s}-I C X}$ $\left(-Y_{1},-Y_{2}\right)$ against $\widetilde{\mathbb{H}}_{1}^{\mathrm{s}}:\left(-X_{1},-X_{2}\right) \npreceq_{\mathrm{s}-\mathrm{ICX}}\left(-Y_{1},-Y_{2}\right)$. To this end, let $\left(X_{11}, X_{12}\right), \ldots,\left(X_{n 1}, X_{n 2}\right)$ and $\left(Y_{11}, Y_{12}\right), \ldots,\left(Y_{m 1}, Y_{m 2}\right)$ be realizations of the 
TABLE 5. Percentages of rejection, as estimated from 1000 replicates, of the tests for the $(1,1)$-concordance ordering hypothesis based on $\Theta_{1,(n, m)}^{(1,1)}$, $\Theta_{2,(n, m)}^{(1,1)}$ and $\Theta_{\infty,(n, m)}^{(1,1)}$ under an autoregressive Gaussian process of order one (upper panel) and a moving-average Gaussian process of order one (bottom panel) when $\tau_{D}=1 / 3$

\begin{tabular}{|c|c|c|c|c|c|c|c|c|c|c|}
\hline \multicolumn{2}{|l|}{ Scenario } & \multicolumn{3}{|c|}{$n=100, m=100$} & \multicolumn{3}{|c|}{$n=100, m=200$} & \multicolumn{3}{|c|}{$n=200, m=200$} \\
\hline Serial parameter & $\tau_{C}$ & $\Theta_{1,(n, m)}^{(1,1)}$ & $\Theta_{2,(n, m)}^{(1,1)}$ & $\Theta_{\infty,(n, m)}^{(1,1)}$ & $\Theta_{1,(n, m)}^{(1,1)}$ & $\Theta_{2,(n, m)}^{(1,1)}$ & $\Theta_{\infty,(n, m)}^{(1,1)}$ & $\Theta_{1,(n, m)}^{(1,1)}$ & $\Theta_{2,(n, m)}^{(1,1)}$ & $\Theta_{\infty,(n, m)}^{(1,1)}$ \\
\hline \multirow{4}{*}{$\theta=0$} & $1 / 3$ & 5.9 & 5.8 & 5.5 & 7.2 & 6.7 & 5.8 & 5.5 & 5.0 & 4.4 \\
\hline & .40 & 18.5 & 18.7 & 14.0 & 25.4 & 24.3 & 16.9 & 31.8 & 28.8 & 18.7 \\
\hline & .60 & 96.1 & 93.8 & 79.0 & 99.2 & 99.0 & 93.8 & 100.0 & 99.9 & 98.0 \\
\hline & $1 / 3$ & 6.4 & 5.5 & 4.3 & 6.8 & 6.6 & 5.2 & 5.7 & 6.1 & 4.2 \\
\hline \multirow[t]{3}{*}{$\theta=1 / 4$} & .40 & 19.2 & 17.4 & 11.1 & 23.8 & 21.9 & 14.9 & 30.6 & 27.3 & 18.9 \\
\hline & .60 & 95.2 & 93.3 & 77.3 & 99.1 & 98.6 & 92.6 & 100.0 & 100.0 & 97.4 \\
\hline & $1 / 3$ & 6.4 & 6.2 & 4.0 & 6.4 & 5.9 & 4.1 & 5.8 & 5.2 & 3.3 \\
\hline \multirow[t]{2}{*}{$\theta=1 / 2$} & .40 & 18.9 & 16.8 & 10.0 & 23.0 & 19.8 & 12.0 & 25.9 & 23.7 & 15.1 \\
\hline & .60 & 91.1 & 88.7 & 63.4 & 97.0 & 95.9 & 84.0 & 99.2 & 99.0 & 91.9 \\
\hline \multirow{4}{*}{$\theta=0$} & $1 / 3$ & 6.0 & 5.7 & 5.0 & 7.6 & 7.0 & 6.4 & 5.7 & 5.5 & 4.0 \\
\hline & .40 & 21.1 & 19.5 & 13.2 & 24.9 & 22.1 & 16.3 & 32.3 & 27.6 & 19.7 \\
\hline & .60 & 96.8 & 95.1 & 79.0 & 99.8 & 99.5 & 94.0 & 100.0 & 99.9 & 98.5 \\
\hline & $1 / 3$ & 6.5 & 6.3 & 4.4 & 5.3 & 4.7 & 3.6 & 7.1 & 7.1 & 4.5 \\
\hline \multirow{3}{*}{$\theta=1 / 4$} & .40 & 20.4 & 17.8 & 11.3 & 23.9 & 22.4 & 14.3 & 29.1 & 25.5 & 17.8 \\
\hline & .60 & 94.8 & 92.3 & 73.9 & 98.7 & 98.0 & 91.6 & 100.0 & 100.0 & 97.4 \\
\hline & $1 / 3$ & 6.1 & 5.8 & 4.7 & 7.9 & 7.7 & 4.0 & 5.0 & 4.5 & 3.7 \\
\hline \multirow[t]{2}{*}{$\theta=1 / 2$} & .40 & 20.4 & 19.6 & 11.0 & 22.1 & 20.8 & 13.3 & 29.2 & 26.7 & 17.4 \\
\hline & .60 & 92.7 & 90.1 & 73.3 & 97.3 & 96.0 & 88.8 & 99.6 & 99.4 & 95.3 \\
\hline \multirow{3}{*}{$\theta=0, \ell_{n}=\ell_{m}=1$} & $1 / 3$ & 5.1 & 4.4 & 3.6 & 5.5 & 5.1 & 3.3 & 3.6 & 3.4 & 2.6 \\
\hline & .40 & 20.8 & 18.5 & 11.8 & 25.9 & 23.0 & 15.9 & 27.4 & 25.2 & 18.8 \\
\hline & .60 & 95.1 & 93.2 & 79.4 & 99.3 & 98.8 & 92.9 & 100.0 & 100.0 & 97.3 \\
\hline
\end{tabular}


strongly stationary $\alpha$-mixing processes $\left(X_{t 1}, X_{t 2}\right)_{t \in \mathbb{Z}}$ and $\left(Y_{t 1}, Y_{t 2}\right)_{t \in \mathbb{Z}}$ both taking values in $\left[a_{1}, b_{1}\right] \times\left[a_{2}, b_{2}\right]$. One then has that $\left(-X_{1},-X_{2}\right) \preceq_{\mathbf{s}-\text { ICX }}\left(-Y_{1},-Y_{2}\right)$ if and only if $\left(1-\widetilde{X}_{1}, 1-\widetilde{X}_{2}\right) \preceq_{\mathbf{s}-\operatorname{ICX}}\left(1-\widetilde{Y}_{1}, 1-\widetilde{Y}_{2}\right)$, where $\widetilde{X}_{j}=\left(X_{j}-a_{j}\right) /\left(b_{j}-\right.$ $\left.a_{j}\right) \in[0,1]$ and $\widetilde{Y}_{j}=\left(Y_{j}-a_{j}\right) /\left(b_{j}-a_{j}\right) \in[0,1]$. It is indeed the case since the mapping from $-X_{j}$ to $1-\widetilde{X}_{j}$ is linear increasing. What it means is that the procedure developed for testing $\preceq_{\mathbf{s}-\ell o}^{\star}$ can be performed on the pairs $\left(\widetilde{X}_{i 1}, \widetilde{X}_{i 2}\right)$ and $\left(\widetilde{Y}_{i 1}, \widetilde{Y}_{i 2}\right)$ instead of the pairs of standardized ranks $\left(\widehat{U}_{i 1}, \widehat{U}_{i 2}\right)$ and $\left(\widehat{V}_{i 1}, \widehat{V}_{i 2}\right)$. However, the multiplier method has to be performed by removing the part involving the partial derivatives.

In order to evaluate how such an alternative procedure performs, some simulations have been made when $\mathbf{s}=(1,1)$ and $\mathbf{s}=(2,2)$ in case the marginal distribution is the Beta(1,3); the latter has a decreasing density, hence is 2concave. For simplicity, only the functional $\mu_{\infty}$ has been considered and the corresponding test statistics is noted $\widetilde{\Theta}_{\infty,(n, m)}^{\mathbf{s}}$. The results are found in Table 6 , where for the sake of comparison, the corresponding results for $\Theta_{\infty,(n, m)}^{\mathbf{s}}$ extracted from Table 3 and Table 4, have been reproduced.

Looking at Table 6 , one first notes that the test based on $\widetilde{\Theta}_{\infty,(n, m)}^{\mathbf{s}}$ holds its $5 \%$ nominal level rather well. However, somewhat surprisingly, the test based on $\Theta_{\infty,(n, m)}^{\mathbf{s}}$ is much more powerful than its counterpart derived under additional assumptions on the marginals. A more detailed investigation of tests of s-increasing convex ordering would be worth of interest. Nevertheless, based on these simulation results, it seems that bringing more information about the marginal distributions do not transfer into a more powerful procedure.

\section{Illustrations on real data}

\subsection{Adaptation of the methodology for stochastic comparisons within the same multivariate population}

The statistical methodology developed in this work can easily be adapted for the comparison of two pairs $\left(X_{j}, X_{k}\right)$ and $\left(X_{j^{\prime}}, X_{k^{\prime}}\right)$ that come as marginals of a $d$ variate random vector $\mathbf{X}=\left(X_{1}, \ldots, X_{d}\right)$ with continuous marginals $F_{1}, \ldots, F_{d}$. If $K:[0,1]^{d} \rightarrow[0,1]$ is the unique copula of $\mathbf{X}$, then $C\left(u_{1}, u_{2}\right)=K\left(\mathbf{u}^{(j k)}\right)$ and $D\left(u_{1}, u_{2}\right)=K\left(\mathbf{u}^{\left(j^{\prime} k^{\prime}\right)}\right)$, where

$$
\mathbf{u}_{\ell}^{(j k)}=\left\{\begin{array}{cc}
u_{1}, & \ell=j \\
u_{2}, & \ell=k \\
1, & \text { otherwise }
\end{array}\right.
$$

In that context, the copula estimators are respectively $C_{n}\left(u_{1}, u_{2}\right)=K_{n}\left(\mathbf{u}^{(j k)}\right)$ and $D_{n}\left(u_{1}, u_{2}\right)=K_{n}\left(\mathbf{u}^{\left(j^{\prime} k^{\prime}\right)}\right)$, where $K_{n}$ is the $d$-dimensional empirical copula computed from $\mathbf{X}_{1}, \ldots, \mathbf{X}_{n}$. According to Bücher and Volgushev (2013), as long as $K$ is regular, i.e. $\dot{K}_{\ell}=\partial K / \partial u_{\ell}$ exists and is continuous on $\left\{\mathbf{u} \in[0,1]^{d}: 0<\right.$ $\left.u_{\ell}<1\right\}$ for each $\ell \in\{1, \ldots, d\}$, and under the same $\alpha$-mixing conditions than 
TABLE 6. Percentages of rejection, as estimated from 1000 replicates, of the tests for the $(1,1)$-concordance ordering (upper panel) and the lower orthant (2,2)-concordance ordering (bottom panel) of $\Theta_{\infty,(n, m)}^{\mathbf{s}}$ and of the modified test based on $\widetilde{\Theta}_{\infty,(n, m)}^{\mathbf{s}}$ under Beta(1,3) marginal distributions

\begin{tabular}{|c|c|c|c|c|c|c|c|c|c|c|}
\hline \multirow[b]{2}{*}{$C$} & \multicolumn{4}{|c|}{ Scenario } & \multicolumn{2}{|c|}{$n=100, m=100$} & \multicolumn{2}{|c|}{$n=100, m=200$} & \multicolumn{2}{|c|}{$n=200, m=200$} \\
\hline & $\tau_{C}$ & $D$ & $\tau_{D}$ & $\Theta_{\infty,(C, D)}^{\mathbf{s}}$ & $\Theta_{\infty,(n, m)}^{\mathbf{s}}$ & $\widetilde{\Theta}_{\infty,(n, m)}^{\mathbf{s}}$ & $\Theta_{\infty,(n, m)}^{\mathbf{s}}$ & $\widetilde{\Theta}_{\infty,(n, m)}^{\mathbf{s}}$ & $\Theta_{\infty,(n, m)}^{\mathbf{s}}$ & $\widetilde{\Theta}_{\infty,(n, m)}^{\mathbf{s}}$ \\
\hline $\mathrm{N}$ & $1 / 3$ & $\mathrm{~N}$ & $1 / 3$ & 0 & 3.6 & 5.0 & 3.3 & 5.9 & 2.6 & 4.3 \\
\hline $\mathrm{N}$ & $2 / 3$ & $\mathrm{~N}$ & $2 / 3$ & 0 & 1.9 & 6.1 & 3.7 & 5.1 & 2.8 & 5.4 \\
\hline $\mathrm{Gu}$ & .40 & $\mathrm{C} \ell$ & $1 / 3$ & .0469 & 23.6 & 14.8 & 39.2 & 15.2 & 55.1 & 18.3 \\
\hline $\mathrm{Gu}$ & .60 & $\mathrm{C} \ell$ & $1 / 3$ & .0861 & 90.2 & 27.1 & 97.9 & 35.7 & 99.7 & 46.0 \\
\hline $\mathrm{Gu}$ & .40 & $\mathrm{C} \ell$ & $2 / 3$ & .0169 & 0.3 & 4.2 & 0.3 & 3.9 & 0.4 & 4.0 \\
\hline $\mathrm{Gu}$ & .60 & $\mathrm{C} \ell$ & $2 / 3$ & .0362 & 9.1 & 9.4 & 16.8 & 10.2 & 29.4 & 8.9 \\
\hline $\mathrm{Gu}$ & .80 & $\mathrm{C} \ell$ & $2 / 3$ & .0615 & 72.5 & 15.3 & 96.0 & 17.6 & 99.1 & 22.1 \\
\hline $\mathrm{Gu}$ & .33 & $\mathrm{C} \ell$ & $1 / 3$ & 0 & 3.3 & 6.7 & 2.2 & 6.7 & 4.7 & 6.6 \\
\hline $\mathrm{Gu}$ & .66 & $\mathrm{C} \ell$ & $2 / 3$ & 0 & 3.0 & 6.7 & 1.5 & 5.9 & 2.9 & 7.7 \\
\hline $\mathrm{Gu}$ & .60 & $\mathrm{C} \ell$ & $1 / 3$ & .0258 & 95.7 & 12.4 & 97.5 & 12.8 & 99.8 & 16.5 \\
\hline $\mathrm{Gu}$ & .80 & $\mathrm{C} \ell$ & $1 / 3$ & .0387 & 100.0 & 14.6 & 100.0 & 16.9 & 100.0 & 21.1 \\
\hline $\mathrm{Gu}$ & .80 & $\mathrm{C} \ell$ & $2 / 3$ & .0080 & 80.8 & 6.0 & 71.6 & 8.2 & 97.8 & 9.1 \\
\hline
\end{tabular}


those in Proposition 4.1, the empirical process $\mathbb{K}_{n}=\sqrt{n}\left(K_{n}-K\right)$ converges weakly in the space $\ell^{\infty}\left([0,1]^{d}\right)$ to a limit of the form

$$
\mathbb{K}(\mathbf{u})=\mathbb{B}_{C}(\mathbf{u})-\sum_{\ell=1}^{d} \dot{K}_{\ell}(\mathbf{u}) \mathbb{B}_{C}\left(\mathbf{u}^{(\ell)}\right)
$$

In this expression, $\mathbb{B}_{C}$ is a Gaussian process on $[0,1]^{d}$ with mean zero such that for $\mathbf{U}_{\ell}=\left(F_{1}\left(X_{\ell 1}\right), \ldots, F_{d}\left(X_{\ell d}\right)\right)$, the covariance function of $\mathbb{B}_{C}$ is

$$
\Gamma_{\mathbb{B}_{C}}\left(\mathbf{u}, \mathbf{u}^{\prime}\right)=\sum_{\ell \in \mathbb{Z}} \operatorname{cov}\left\{\mathbb{I}\left(\mathbf{U}_{0} \leq \mathbf{u}\right), \mathbb{I}\left(\mathbf{U}_{\ell} \leq \mathbf{u}^{\prime}\right)\right\}
$$

One can then derive an adapted version of Proposition 4.1. A multiplier version of $\mathbb{K}_{n}$ based on a serial multiplier sample $\boldsymbol{\xi}$ can be defined in the same line as those for $\mathbb{C}_{n}$ and $\mathbb{D}_{m}$; the counterpart of Proposition 5.1 is straightforward to obtain. From an implementation perspective, the only necessary adjustment consists in using the same multiplier sample $\boldsymbol{\xi}$ for both datasets of $n$ pairs.

\subsection{Cook E Johnson's Uranium exploration data}

The Uranium exploration dataset has been first considered by Cook and Johnson (1981, 1986). It consists of concentrations of seven chemical elements measured on $n=655$ water samples collected from the Montrose quadrangle of western Colorado (USA). All these samples are independent from each other. The following analyses will focus on four of these variables, namely Potassium $(\mathrm{K})$, Caesium (Cs), Scandium (Sc) and Titanium (Ti).

The histograms and the scatterplots, both of the original data $\mathbf{X}_{1}, \ldots, \mathbf{X}_{655}$ and of the standardized ranks $\widehat{\mathbf{U}}_{1}, \ldots, \widehat{\mathbf{U}}_{655}$, are found in Figure 1. Looking at the histograms of the four variables, it is clear that they are marginally quite different. Hence, if the goal is to perform stochastic comparisons among some of the pairs, it cannot reasonably be assumed that they belong to the same Fréchet class. The s-concordance orderings, which assume nothing on the marginal distributions (apart from being continuous, which is the case here), are therefore well-suited for these data.

The first analysis concerns the stochastic comparison of ( $\mathrm{Cs}, \mathrm{Ti}$ ) with (K,Cs) using the adapted methodology of subsection 7.1 with $\mathcal{K}=25$ and $B=10,000$ i.i.d. multiplier samples; the estimation of the partial derivatives is done by letting $b=1$, since the tests performed with $b=3$ yielded very similar values. If one looks at Figure 1 , it seems that $(\mathrm{Cs}, \mathrm{Ti}) \preceq_{(1,1)-\ell o}^{*}(\mathrm{~K}, \mathrm{Cs})$ cannot hold; this is confirmed by the results of the tests that are found in Table 7 when $\mathbf{s}=(1,1)$. This is also confirmed, to a certain extent, by the values of the empirical Kendall's tau, namely $\tau_{n}(\mathrm{Cs}, \mathrm{Ti})=.279$ and $\tau_{n}(\mathrm{~K}, \mathrm{Cs})=.200$.

Nevertheless, their dependence structures can somewhat be ordered if one looks at other levels. Hence, while the ordering $\preceq_{(2,1)-\ell o}^{\star}$ is still rejected, the null hypotheses $\mathbb{H}_{0}^{(1,2)}$ and $\mathbb{H}_{0}^{(2,2)}$ are accepted at the $5 \%$ level by the tests based 

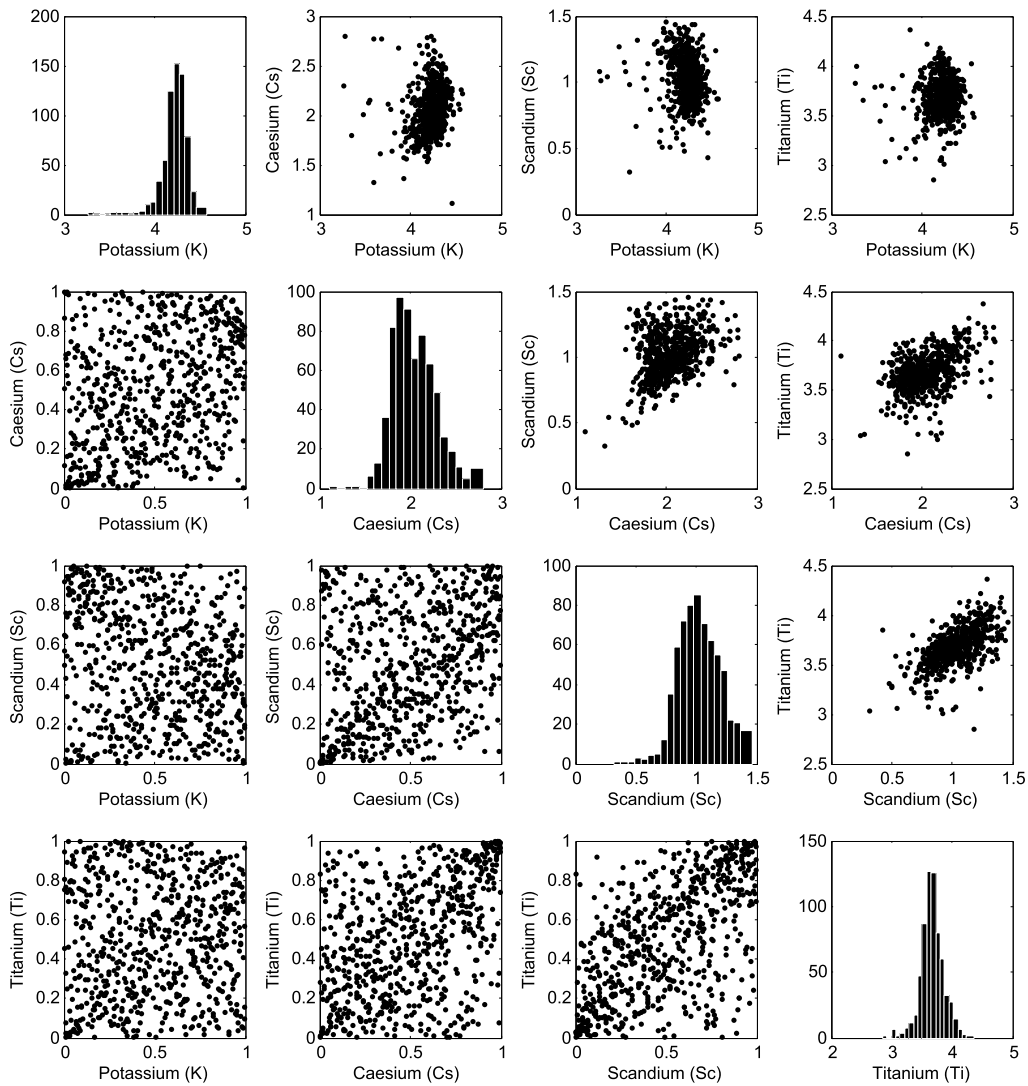

FIG 1. Histograms (diagonal) and scatterplots of the original data (upper triangle) and of their associated standardized ranks (lower triangle) for chemical elements $K, C s, S c$ and Ti in the Uranium exploratory dataset

TABLE 7

P-values (in \%) as estimated with $B=10,000$ multiplier bootstrap samples for the stochastic comparisons of pairs in the Uranium exploratory dataset

\begin{tabular}{cc|ccc}
\hline Comparison & $\mathbf{s}$ & $\Theta_{1,(n, m)}^{(\mathbf{s})}$ & $\Theta_{2,(n, m)}^{(\mathbf{s})}$ & $\Theta_{\infty,(n, m)}^{(\mathbf{s})}$ \\
\hline \multirow{3}{*}{$(\mathrm{Cs}, \mathrm{Ti}) \preceq_{\mathbf{s}-\ell_{o}}^{\star}(\mathrm{K}, \mathrm{Cs})$} & $(1,1)$ & .4 & .1 & $<.001$ \\
& $(2,1)$ & 2.1 & .8 & .001 \\
& $(1,2)$ & 11.4 & 5.1 & .2 \\
& $(2,2)$ & 10.9 & 10.0 & 1.0 \\
\hline & $(1,1)$ & $<.001$ & $<.001$ & $<.001$ \\
$(\mathrm{Cs}, \mathrm{Ti}) \preceq_{\mathbf{s}-\ell o}^{\star}(\mathrm{Cs}, \mathrm{Sc})$ & $(2,1)$ & $<.001$ & $<.001$ & .7 \\
& $(1,2)$ & 8.7 & 4.1 & .1 \\
\hline
\end{tabular}

on the functionals $\mu_{1}$ and $\mu_{2}$; these hypotheses are however rejected by the test based on $\mu_{\infty}$. In view of the link between the lower orthant $(2,2)$-concordance ordering and conditional Spearman's rho established in subsection 3.3, this sug- 
gests that the dependence level of (K,Cs), as measured by Spearman's rho, can be larger than that of (Cs, Ti), and vice versa, when one restricts to some lower corners of $[0,1]^{2}$. Replacing $C$ with $C_{n}$ and developing formula (8) yields

$$
\widehat{\rho}_{u_{k_{1}}, u_{k_{2}}}^{\mathrm{Sp}}\left(X_{1}, X_{2}\right)=\frac{12 Z_{k_{1}, k_{2}}^{(2,2)}-3 u_{k_{1}}^{2} u_{k_{2}}^{2}}{2\left(u_{k_{1}} \wedge u_{k_{2}}\right)\left\{3 u_{k_{1}} u_{k_{2}}-\left(u_{k_{1}} \wedge u_{k_{2}}\right)\right\}-3 u_{k_{1}}^{2} u_{k_{2}}^{2}}
$$

as an empirical Spearman's rho conditioned on $\left[0, u_{k_{1}}\right] \times\left[0, u_{k_{2}}\right]$, with $u_{k}=$ $(k-1 / 2) / \mathcal{K}$. The top panel of Figure 2 shows $\widehat{\rho}_{u_{k_{1}}, u_{k_{2}}}^{\mathrm{Sp}}(\mathrm{K}, \mathrm{Cs})-\widehat{\rho}_{u_{k_{1}}, u_{k_{2}}}^{\mathrm{Sp}}(\mathrm{Cs}, \mathrm{Ti})$. It can be seen that for $u_{1} \leq .7$, say, Spearman's rho is larger for (K,Cs) compared to $(\mathrm{Cs}, \mathrm{Ti}$ ), often significantly (curve above 0 ); in the complementary region, it is for (Cs, Ti) that Spearman's rho is larger (curve below 0), but by a much less amount. These features could have been anticipated from the results of the tests when $\mathbf{s}=(2,2)$. That the test based on $\mu_{\infty}$ has rejected the null hypothesis of a $(2,2)$-concordance ordering may be explained by the fact that this functional can be strongly influenced by local discrepancies, while $\mu_{1}, \mu_{2}$ are global distances.
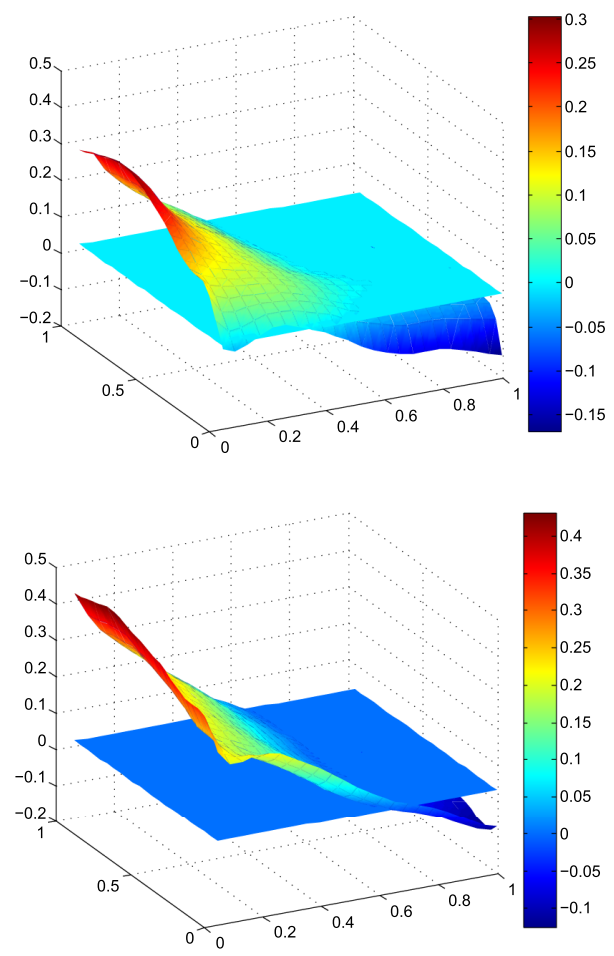

FIG 2. 3d-Plots of the difference between conditional Spearman's rho of (K,Cs) and (Cs, Ti) (top) and of the difference between conditional Spearman's rho of (K, Cs) and (Cs, Ti) (bottom) in the Uranium exploratory dataset

The pair $(\mathrm{Cs}, \mathrm{Ti})$ has also been stochastically compared to $(\mathrm{Cs}, \mathrm{Sc})$; the results in Table 7 are similar to those of the previous analysis. Thus, while the null 
hypothesis $(\mathrm{Cs}, \mathrm{Ti}) \preceq_{(1,1)-\ell_{o}}^{\star}(\mathrm{Cs}, \mathrm{Sc})$ is clearly rejected, there is nevertheless some sort of ordering at the level of conditional measures of association like Spearman's rho that can be clearly observed on the bottom panel of Figure 2.

\subsection{Evolution of exchange rates}

Another illustration concerns the $n=228$ exchange rates of the Euro (EUR), Canada (CAN), Australia (AUS), New Zealand (NZE) and Japan (JAP) currencies as measured monthly with respect to US dollar between January 1999 and December 2017. The five time series are found at the top of Figure 3 (Japan currency has been divided by 100). The series are clearly not marginally stationary; however, since the lag-1 differentiated series are reasonably stationary (see bottom of Figure 3), the latter will be considered for the upcoming analyses.
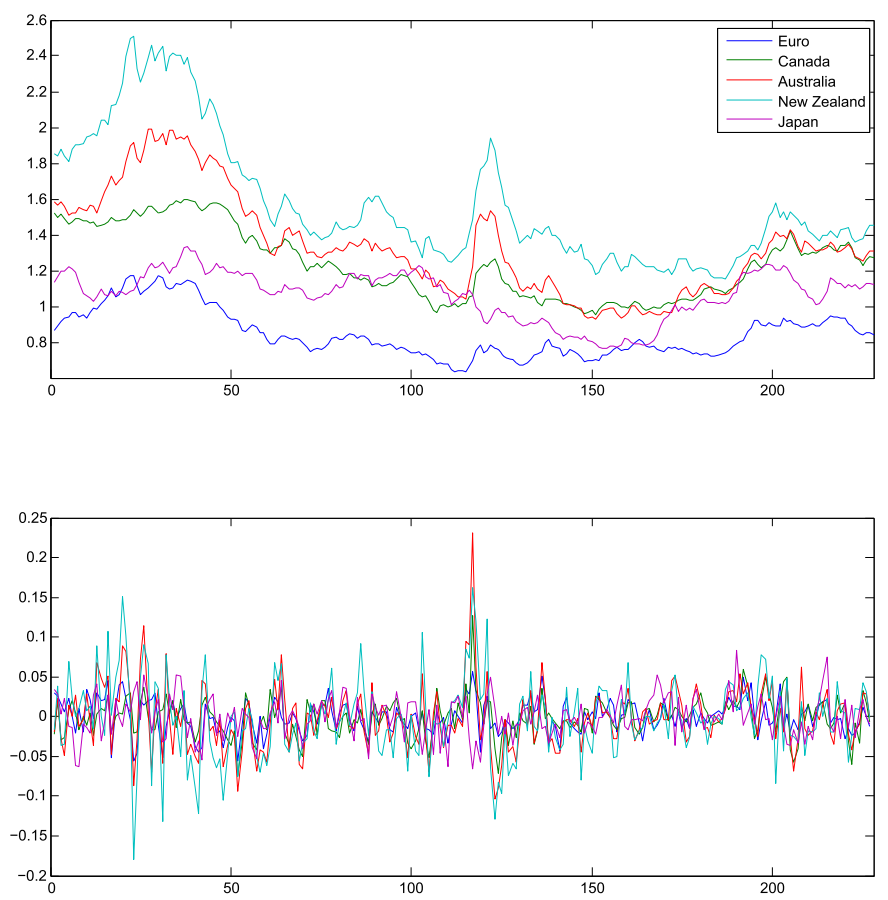

FIG 3. Time series of the exchange rates of Euro, Canada, Australia, New Zealand and Japan currencies with respect to US dollard between January 1999 and December 2017 (top) and lag-1 differentiated series (bottom)

A look at the scatterplots of the lag-1 differentiated time series shown in the lower triangle of Figure 4 indicates a possible radial symmetry structure; this is confirmed by the test of radial symmetry of Bahraoui and Quessy (2017), where based on 1,000 multiplier samples, the test's p-value is estimated to $21,2 \%$; note however that the test assumes serial independence (to date, no test exists to 
deal with that situation). Radial symmetry means that the orderings $\preceq_{\mathbf{s}-\ell o}^{\star}$ and $\preceq_{\mathbf{s}-u o}^{\star}$ between two-pairs are equivalent. Because the relationship of Japan with other currencies is quite low, except maybe with the Australian currency, the former has been excluded of the following analysis; these low dependence levels can be seen from the values of Kendall's tau, i.e. $\tau_{n}(\mathrm{EUR}, \mathrm{JAP})=0.341$, $\tau_{n}(\mathrm{CAN}, \mathrm{JAP})=0.152, \tau_{n}(\mathrm{AUS}, \mathrm{JAP})=0.226$ and $\tau_{n}(\mathrm{NZE}, \mathrm{JAP})=0.165$.
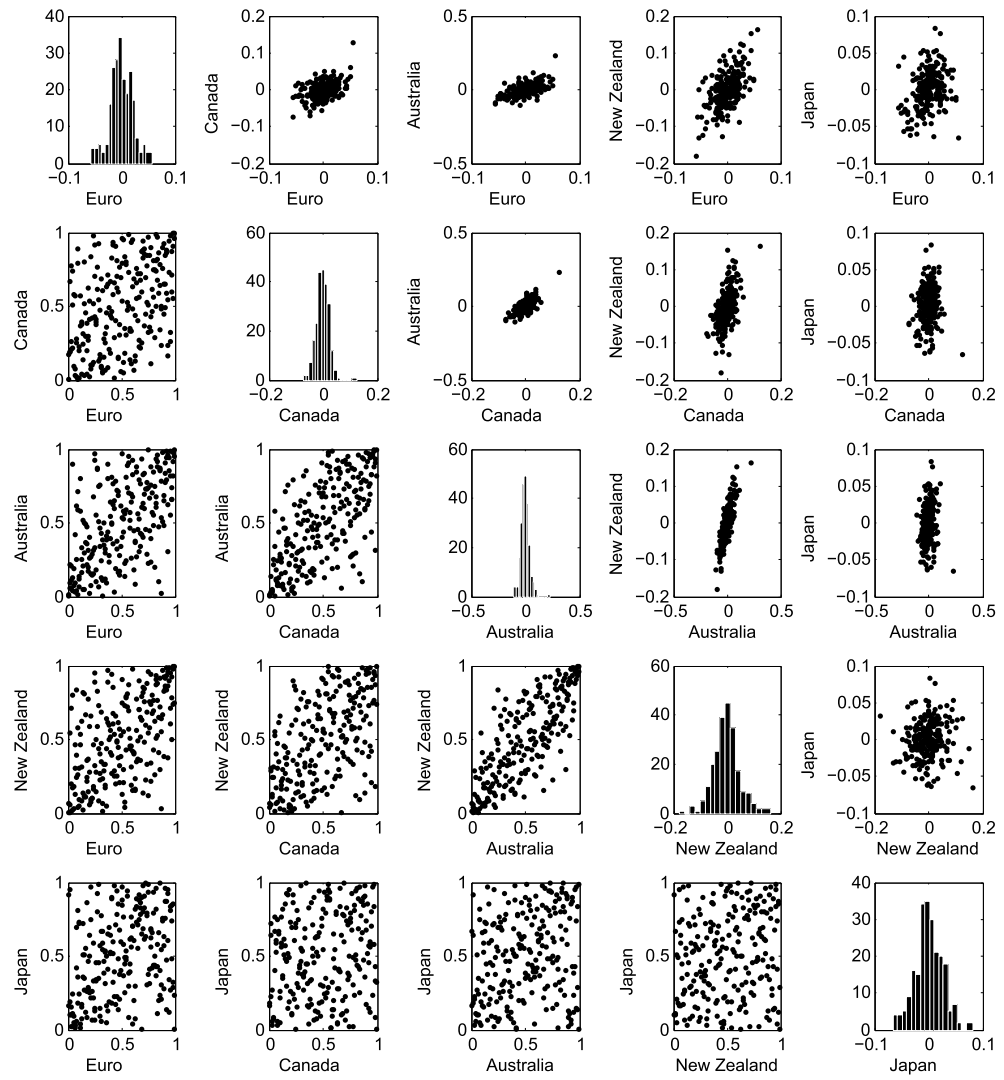

FIG 4. Histograms (diagonal) and scatterplots of the original data (upper triangle) and of their associated standardized ranks (lower triangle) for the lag-1 differentiated exchange rates of five currencies (Euro, Canada, Australia, New Zealand and Japan) with respect to US dollar between January 1999 and December 2017

The results of the test based on $\Theta_{\infty,(n, m)}^{(\mathbf{s})}$ for each of the six possible comparisons of non-overlapping pairs of (EUR,CAN,AUS,NZE) are presented in Table 8. Here, the number of serial multiplier samples, as described in subsection 6.4, has been set to $B=1,000$ with $\ell_{n}=\left\lfloor 1.1 \times 228^{1 / 4}\right\rfloor=4$. One of the conclusions is that the pair (AUS,NZE) significantly dominates (EUR,CAN) at the $5 \%$ level according to $(1,1)$-concordance; this feature was expected from the respective scatterplots of standardized ranks in Figure 4. A similar conclu- 
sion can be made about the dominance of (CAN,NZE) over (EUR,AUS), and of (CAN,AUS) over (EUR,NZE); in these cases, however, the use of a formal test prove crucial, since the conclusion could hardly be based on looking at the scatterplots only.

\begin{tabular}{|c|c|c|c|c|c|}
\hline$X$ & $Y$ & $\preceq_{(1,1)-\ell_{o}}^{\star}$ & $\preceq_{(2,1)-\ell o}^{\star}$ & $\preceq_{(1,2)-\ell o}^{\star}$ & $\varliminf_{(2,2)-\ell_{o}}^{\star}$ \\
\hline$\overline{\text { (EUR,CAN) }}$ & (AUS,NZE) & 100 & 100 & 100 & 96.3 \\
\hline (EUR,AUS) & (CAN,NZE) & 56,4 & 35.1 & 43.5 & 35.5 \\
\hline (EUR,NZE) & (CAN,AUS) & 98.0 & 91.7 & 95.5 & 94.2 \\
\hline (CAN,AUS) & (EUR,NZE) & 4.3 & 6.9 & 1.5 & 2.7 \\
\hline (CAN,NZE) & (EUR,AUS) & 2.9 & 4.5 & 4.2 & 2.3 \\
\hline (AUS,NZE) & (EUR,CAN) & $<.001$ & $<.001$ & $<.001$ & $<.001$ \\
\hline
\end{tabular}

\section{Discussion}

In this paper, a new family of stochastic orders that allow for marginal-free comparisons between random pairs have been introduced; these orders generalize the usual concordance ordering. The construction of this hierarchical family of orders is rooted around the concept of s-increasing convex orders computed at the level of the copula that uniquely characterizes the dependence in a random couple. It has been shown, in particular, how these orders are related to Spearman's measure of association. Also, a complete set of statistical tools has been developed to formally assess the stochastic dominance of a random pair on another pair; the proposed framework is quite general, as it allows for serially dependent data, and can also accommodate the case when the two pairs are subvectors drawn from the same multivariate population.

The analysis performed on the classical Uranium exploration data is typical of the information that can be extracted in a multivariate dataset when looking from the point-of-view of s-concordance orders. Hence, while the proposed statistical methodology has clearly discarded the usual concordance ordering hypothesis for being too strong, it allowed to establish a relationship at the level of the less restrictive order $\mathbf{s}=(2,2)$; in turn, this can be interpreted as the dominance of one pair on another at the level of conditional Spearman's rho when one restricts to some lower corners of $[0,1]$.

In a future investigation, it would be interesting to generalize the notions of positive quadrant dependence (PQD) and negative quadrant dependence (NQD) with respect to the new class of s-concordance orderings. Specifically, one could define $\left(X_{1}, X_{2}\right)$ to be s-PQD (resp. s-NQD) if $\left(X_{1}^{\perp}, X_{2}^{\perp}\right) \preceq_{\mathbf{s}-\ell o}^{\star}\left(X_{1}, X_{2}\right)$ (resp. $\left.\left(X_{1}, X_{2}\right) \preceq_{\mathbf{s}-\ell o}^{\star}\left(X_{1}^{\perp}, X_{2}^{\perp}\right)\right)$, where $\left(X_{1}^{\perp}, X_{2}^{\perp}\right)$ is a copy of $\left(X_{1}, X_{2}\right)$, but with independent components. The statistical tools of Section 4 and Section 5 could then be adapted in order to provide not only interesting extensions of the tests of Scaillet (2005) and Gijbels, Omelka and Sznajder (2010) to s-PQD and s-NQD, 
but also provide new test statistics that are valid under serial data and/or when the pairs are from the same multivariate population.

Another fruitful avenue of research would be to design an alternative bootstrap procedure in order to ensure an exact asymptotic size for the tests. A promising way would be to adapt to the current context a bootstrap procedure proposed by Linton, Song and Whang (2010) for testing univariate stochastic dominance that improves the power of the tests by Barrett and Donald (2003). Such a version for the tests developed in this work is, however, far from being straightforward. In fact, since one is working at the level of copulas, the complexity of the asymptotics is increased due to the use of ranks. In addition, the methodology would have to be adapted to serial data.

Appendix A: Proofs of Properties 2.1, 2.2, 2.3 and 2.4

\section{A.1. Proof of Property 2.1}

For $j=1,2$, let $\bar{F}_{j}^{\perp}(x)=\mathbb{P}\left(-X_{j}>x\right)=\mathbb{P}\left(X_{j}<-x\right)=F_{j}(-x)$; similarly, let $G_{j}^{\perp}(x)=G_{j}(-x)$. By definition, $\left(-X_{1},-X_{2}\right) \preceq_{\mathbf{s}-\ell_{o}}^{\star}\left(-Y_{1},-Y_{2}\right)$ if and only if $\left(\bar{F}_{1}^{\perp}\left(-X_{1}\right), \bar{F}_{2}^{\perp}\left(-X_{2}\right)\right) \preceq_{\text {s-ICX }}\left(\bar{G}_{1}^{\perp}\left(-Y_{1}\right), \bar{G}_{2}^{\perp}\left(-Y_{2}\right)\right)$. This is equivalent to $\left(F_{1}\left(X_{1}\right), F_{2}\left(X_{2}\right)\right) \preceq_{\text {s-ICX }}\left(G_{1}\left(Y_{1}\right), G_{2}\left(Y_{2}\right)\right)$, which is exactly the definition of $\left(X_{1}, X_{2}\right) \preceq_{\mathbf{s}-u o}^{\star}\left(Y_{1}, Y_{2}\right)$.

\section{A.2. Proof of Property 2.2}

Given that $\preceq_{\mathbf{s}-\mathrm{ICX}}$ is a hierarchic order, see Equation (2.21) of Denuit, Lefèvre and Mesfioui (1999), the proof is straightforward.

\section{A.3. Proof of Property 2.3}

(i) First define $F_{j}^{\psi}(x)=\mathbb{P}\left\{\psi\left(X_{j}\right) \leq x\right\}$ for $j \in\{1,2\}$. When $\psi_{j}$ is strictly increasing, it follows easily that $F_{j}^{\psi_{j}}\left\{\psi_{j}\left(X_{j}\right)\right\}=F_{j}\left(X_{j}\right)$ and $\bar{F}_{j}^{\psi_{j}}\left\{\psi_{j}\left(X_{j}\right)\right\}=$ $\bar{F}_{j}\left(X_{j}\right)$, so that Definition 2.1 entails that $\left(\psi_{1}\left(X_{1}\right), \psi_{2}\left(X_{2}\right)\right) \preceq_{\mathbf{s}}^{\star}\left(\psi_{1}\left(Y_{1}\right), \psi_{2}\left(Y_{2}\right)\right)$ if and only if $\left(X_{1}, X_{2}\right) \preceq_{\mathrm{s}}^{\star}\left(Y_{1}, Y_{2}\right)$. This equivalence means that the s-concordance ordering of two pairs depends only on their respective copulas. The remaining of the proof is therefore straightforward, upon noting that:

(ii) when both $\psi_{1}$ and $\psi_{2}$ are decreasing, the copula of $\left(\psi_{1}\left(X_{1}\right), \psi_{2}\left(X_{2}\right)\right)$ is the same as that of $\left(-X_{1},-X_{2}\right)$.

(iii) when $\psi_{1}$ is increasing and $\psi_{2}$ is decreasing, the copula of $\left(\psi_{1}\left(X_{1}\right), \psi_{2}\left(X_{2}\right)\right)$ is the same as that of $\left(X_{1},-X_{2}\right)$.

(iv) when $\psi_{1}$ is decreasing and $\psi_{2}$ is increasing, the copula of $\left(\psi_{1}\left(X_{1}\right), \psi_{2}\left(X_{2}\right)\right)$ is the same as that of $\left(-X_{1}, X_{2}\right)$. 


\section{A.4. Proof of Property 2.4}

If $C$ is radially symmetric, $\left(\bar{F}_{1}\left(X_{1}\right), \bar{F}_{2}\left(X_{2}\right)\right)$ and $\left(F_{1}\left(X_{1}\right), F_{2}\left(X_{2}\right)\right)$ are equal in distribution. As a consequence,

$$
\begin{aligned}
\left(X_{1}, X_{2}\right) \preceq_{\mathbf{s}-\ell o}^{\star}\left(Y_{1}, Y_{2}\right) & \Longleftrightarrow\left(\bar{F}_{1}\left(X_{1}\right), \bar{F}_{2}\left(X_{2}\right)\right) \preceq_{\mathbf{s}-\mathrm{ICX}}\left(\bar{G}_{1}\left(Y_{1}\right), \bar{G}_{2}\left(Y_{2}\right)\right) \\
& \Longleftrightarrow\left(F_{1}\left(X_{1}\right), F_{2}\left(X_{2}\right)\right) \preceq_{\mathbf{s}-\mathrm{ICX}}\left(G_{1}\left(Y_{1}\right), G_{2}\left(Y_{2}\right)\right) \\
& \Longleftrightarrow\left(X_{1}, X_{2}\right) \preceq_{\mathbf{s}-u o}^{\star}\left(Y_{1}, Y_{2}\right) .
\end{aligned}
$$

\section{Appendix B: Proofs of Propositions 2.1, 2.2, 4.1 and 5.1}

\section{B.1. Proof of Proposition 2.1}

The proof turns around Property 4.1 of Denuit, Lefèvre and Mesfioui (1999) that states that if $\phi_{1}$ is $s_{1}$-convex and $\phi_{2}$ is $s_{2}$-convex, then $\left(X_{1}, X_{2}\right) \preceq_{\mathbf{s}-\text { ICX }}\left(Y_{1}, Y_{2}\right)$ entails $\left(\phi_{1}\left(X_{1}\right), \phi_{2}\left(X_{2}\right)\right) \preceq_{\mathbf{s}-\mathrm{ICX}}\left(\phi_{1}\left(Y_{1}\right), \phi_{2}\left(Y_{2}\right)\right)$.

\section{Lower orthant s-concordance}

(i) By definition, $\left(X_{1}, X_{2}\right) \preceq_{\mathbf{s}-\ell_{o}}^{\star}\left(Y_{1}, Y_{2}\right)$ means that $\left(\bar{F}_{1}\left(X_{1}\right), \bar{F}_{2}\left(X_{2}\right)\right) \preceq_{\mathbf{s}-\text { ICX }}$ $\left(\bar{F}_{1}\left(Y_{1}\right), \bar{F}_{2}\left(Y_{2}\right)\right)$. Since by assumption, $F_{j}^{-1}$ is $s_{j}$-concave, then $-\bar{F}_{j}^{-1}(x)=$ $-F_{j}^{-1}(1-x)$ is $s_{j}$-convex. One concludes that $\left(-X_{1},-X_{2}\right) \preceq_{\mathbf{s}-\mathrm{ICX}}\left(-Y_{1},-Y_{2}\right)$.

(ii) Since $F_{j}$ is $s_{j}$-concave, $\bar{F}_{j}(-x)$ is $s_{j}$-convex so that $\left(-X_{1},-X_{2}\right) \preceq$ s-ICX $\left(-Y_{1},-Y_{2}\right)$ entails $\left(\bar{F}_{1}\left(X_{1}\right), \bar{F}_{2}\left(X_{2}\right)\right) \preceq_{\text {s-ICX }}\left(\bar{F}_{1}\left(Y_{1}\right), \bar{F}_{2}\left(Y_{2}\right)\right)$, or equivalently $\left(X_{1}, X_{2}\right) \preceq_{\mathbf{s}-\ell o}^{\star}\left(Y_{1}, Y_{2}\right)$.

$\underline{\text { Upper orthant s-concordance }}$

(iii) By definition, $\left(X_{1}, X_{2}\right) \preceq_{\mathbf{s}-u o}^{\star}\left(Y_{1}, Y_{2}\right)$ means that $\left(F_{1}\left(X_{1}\right), F_{2}\left(X_{2}\right)\right) \preceq_{\mathbf{s}-\text { ICX }}$ $\left(F_{1}\left(Y_{1}\right), F_{2}\left(Y_{2}\right)\right)$. The $s_{j}$-convexity of $F_{j}^{-1}$ then entails $\left(X_{1}, X_{2}\right) \preceq_{\mathbf{s}-\mathrm{ICX}}\left(Y_{1}, Y_{2}\right)$.

(iv) Since $F_{j}$ is $s_{j}$-convex, $\left(X_{1}, X_{2}\right) \preceq_{\text {s-ICX }}\left(Y_{1}, Y_{2}\right)$ entails $\left(F_{1}\left(X_{1}\right), F_{2}\left(X_{2}\right)\right)$ $\preceq_{\text {s-ICX }}\left(F_{1}\left(Y_{1}\right), F_{2}\left(Y_{2}\right)\right)$, i.e. $\left(X_{1}, X_{2}\right) \preceq_{\mathbf{s}-u o}^{\star}\left(Y_{1}, Y_{2}\right)$.

\section{B.2. Proof of Proposition 2.2}

Let $u_{1}, u_{2}, a_{1}, a_{2} \in[0,1]$ and define, for $\mathbf{i}=\left(i_{1}, i_{2}\right) \in \mathbb{N}^{2}$ and $x_{+}=\max (x, 0)$,

$$
\mathcal{O}_{\mathbf{i}}\left(a_{1}, a_{2} ; u_{1}, u_{2}\right)=\frac{\left(u_{1}-a_{1}\right)_{+}^{i_{1}-1}}{\left(i_{1}-1\right) !} \times \frac{\left(u_{2}-a_{2}\right)_{+}^{i_{2}-1}}{\left(i_{2}-1\right) !} .
$$

Letting $g\left(a_{1}, a_{2}\right)=\mathbb{I}\left(a_{1} \leq u_{1}, a_{2} \leq u_{2}\right)$, one can show by induction that

$$
\mathcal{O}_{\mathbf{i}}\left(a_{1}, a_{2} ; u_{1}, u_{2}\right)=\mathcal{J}_{\mathbf{i}}\left(u_{1}, u_{2} ; g\right) .
$$


Now since $\left(X_{1}, X_{2}\right) \preceq_{\mathbf{s}-\ell_{o}}^{\star}\left(Y_{1}, Y_{2}\right)$ holds if and only if $\left(\bar{F}_{1}\left(X_{1}\right), \bar{F}_{2}\left(X_{2}\right)\right) \preceq_{\mathbf{s}-\text { ICX }}$ $\left(\bar{G}_{1}\left(Y_{1}\right), \bar{G}_{2}\left(Y_{2}\right)\right)$, Characterization 3.1 of Denuit, Lefèvre and Mesfioui (1999) for the s-increasing convex order may be formulated as

$$
\begin{aligned}
& \mathrm{E}\left\{\mathcal{O}_{\mathbf{s}}\left(u_{1}, u_{2} ; \bar{F}_{1}\left(X_{1}\right), \bar{F}_{2}\left(X_{2}\right)\right)\right\} \leq \mathrm{E}\left\{\mathcal{O}_{\mathbf{s}}\left(u_{1}, u_{2} ; \bar{G}_{1}\left(Y_{1}\right), \bar{G}_{2}\left(Y_{2}\right)\right)\right\}, \\
& \mathrm{E}\left\{\mathcal{O}_{\left(i_{1}, s_{2}\right)}\left(0, u_{2} ; \bar{F}_{1}\left(X_{1}\right), \bar{F}_{2}\left(X_{2}\right)\right)\right\} \leq \mathrm{E}\left\{\mathcal{O}_{\left(i_{1}, s_{2}\right)}\left(0, u_{2} ; \bar{G}_{1}\left(Y_{1}\right), \bar{G}_{2}\left(Y_{2}\right)\right)\right\} \forall i_{1}<s_{1}, \\
& \mathrm{E}\left\{\mathcal{O}_{\left(s_{1}, i_{2}\right)}\left(u_{1}, 0 ; \bar{F}_{1}\left(X_{1}\right), \bar{F}_{2}\left(X_{2}\right)\right)\right\} \leq \mathrm{E}\left\{\mathcal{O}_{\left(s_{1}, i_{2}\right)}\left(u_{1}, 0 ; \bar{G}_{1}\left(Y_{1}\right), \bar{G}_{2}\left(Y_{2}\right)\right)\right\} \forall i_{2}<s_{2}, \\
& \mathrm{E}\left\{\mathcal{O}_{\mathbf{i}}\left(0,0 ; \bar{F}_{1}\left(X_{1}\right), \bar{F}_{2}\left(X_{2}\right)\right)\right\} \leq \mathrm{E}\left\{\mathcal{O}_{\mathbf{i}}\left(0,0 ; \bar{G}_{1}\left(Y_{1}\right), \bar{G}_{2}\left(Y_{2}\right)\right)\right\} \forall \mathbf{i}<\mathbf{s} .
\end{aligned}
$$

Since $\mathcal{O}_{\mathbf{i}}\left(u_{1}, u_{2} ; \bar{F}_{1}\left(X_{1}\right), \bar{F}_{2}\left(X_{2}\right)\right)=\mathcal{O}_{\mathbf{i}}\left(F_{1}\left(X_{1}\right), F_{2}\left(X_{2}\right), 1-u_{1}, 1-u_{2}\right)$ for any $\mathbf{i} \leq$ $\mathbf{s}, \mathrm{E}\left\{\mathcal{O}_{\mathbf{i}}\left(u_{1}, u_{2} ; \bar{F}_{1}\left(X_{1}\right), \bar{F}_{2}\left(X_{2}\right)\right)\right\}=\mathrm{E}\left\{\mathcal{O}_{\mathbf{i}}\left(F_{1}\left(X_{1}\right), F_{2}\left(X_{2}\right) ; 1-u_{1}, 1-u_{2}\right)\right\}=$ $\mathcal{J}_{\mathbf{i}}\left(1-u_{1}, 1-u_{2} ; C\right)$. From the linearity of the integral, $\mathcal{J}_{\mathbf{i}}(\cdot, \cdot ; C)-\mathcal{J}_{\mathbf{i}}(\cdot, \cdot ; D)=$ $\mathcal{J}_{\mathbf{i}}(\cdot, \cdot ; C-D)$, so that the inequalities in (16) can be written as

$$
\begin{aligned}
\mathcal{J}_{\mathbf{s}}\left(u_{1}, u_{2} ; C-D\right) \leq 0, & \\
\mathcal{J}_{\left(i_{1}, s_{2}\right)}\left(1, u_{2} ; C-D\right) \leq 0, & \text { for } i_{1}<s_{1}, \\
\mathcal{J}_{\left(s_{1}, i_{2}\right)}\left(u_{1}, 1 ; C-D\right) \leq 0, & \text { for } i_{2}<s_{2}, \\
\mathcal{J}_{\left(i_{1}, i_{2}\right)}(1,1 ; C-D) \leq 0, & \text { for } \mathbf{i}<\mathbf{s} .
\end{aligned}
$$

Since $\mathcal{J}_{\left(1, i_{2}\right)}\left(1, u_{2} ; C-D\right)=\mathcal{J}_{\left(i_{1}, 1\right)}\left(u_{1}, 1 ; C-D\right)=0$, the latter can be written as in (6). For the characterization of the upper orthant s-concordance ordering, observe that $\left(X_{1}, X_{2}\right) \preceq_{\mathbf{s}-u o}^{\star}\left(Y_{1}, Y_{2}\right)$ if and only if $\left(-X_{1},-X_{2}\right) \preceq_{\mathrm{s}-\ell o}^{\star}\left(-Y_{1},-Y_{2}\right)$, and that the copulas of $\left(-X_{1},-X_{2}\right)$ and $\left(-Y_{1},-Y_{2}\right)$ are, respectively, $\widehat{C}$ and $\widehat{D}$.

\section{B.3. Proof of Proposition 4.1}

Under the conditions stated, Bücher and Volgushev (2013) obtained the weak convergence in $\ell^{\infty}\left([0,1]^{2}\right)$ of $\mathbb{C}_{n}=\sqrt{n}\left(C_{n}-C\right)$ to $\mathbb{C}$; similarly, $\mathbb{D}_{m}=\sqrt{m}\left(D_{m}-\right.$ $D)$ converges weakly to $\mathbb{D}$. Strictly speaking, because the two samples are independent, these two convergences are simultaneous, i.e. the pair of processes $\left(\mathbb{C}_{n}, \mathbb{D}_{m}\right)$ converges to $(\mathbb{C}, \mathbb{D})$. From there,

$$
\begin{aligned}
\mathbb{L}_{n, m}^{(\mathbf{i})} & =\sqrt{\frac{n m}{n+m}}\left\{\mathcal{J}_{\mathbf{i}}\left(\cdot, \cdot ; C_{n}-D_{m}\right)-\mathcal{J}_{\mathbf{i}}(\cdot, \cdot ; C-D)\right\} \\
& =\sqrt{\frac{n m}{n+m}}\left\{\mathcal{J}_{\mathbf{i}}\left(\cdot, \cdot ; \frac{\mathbb{C}_{n}}{\sqrt{n}}-\frac{\mathbb{D}_{m}}{\sqrt{m}}\right)\right\} \\
& =\mathcal{J}_{\mathbf{i}}\left(\cdot, \cdot ; \sqrt{\frac{m}{n+m}} \mathbb{C}_{n}-\sqrt{\frac{n}{n+m}} \mathbb{D}_{m}\right) \\
& =\mathcal{J}_{\mathbf{i}}\left(\cdot, \cdot ; \sqrt{1-\omega_{n, m}} \mathbb{C}_{n}-\sqrt{\omega_{n, m}} \mathbb{D}_{m}\right)
\end{aligned}
$$

where $\omega_{n, m}=n /(n+m)$. Since the operator $\mathcal{J}_{\mathbf{i}}$ is continuous, one can then conclude that $\mathbb{L}_{n, m}^{(\mathbf{i})} \rightsquigarrow \mathbb{L}^{(\mathbf{i})}=\mathcal{J}_{\mathbf{i}}(\cdot, \cdot ; \sqrt{1-\omega} \mathbb{C}-\sqrt{\omega} \mathbb{D})$. That this convergence happens jointly for any $\mathbf{i} \in \mathbb{N}^{2}$ is obvious from the definition of $\mathbb{L}_{n, m}^{(\mathbf{i})}$ as a functional of $C_{n}-D_{m}$. 


\section{B.4. Proof of Proposition 5.1}

Under the conditions stated, Bücher and Ruppert (2013) obtained that $\left(\mathbb{C}_{n}, \widehat{\mathbb{C}}_{n}\right)$ converges weakly to $(\mathbb{C}, \widetilde{\mathbb{C}})$, where $\widetilde{\mathbb{C}}$ is an independent copy of $\mathbb{C}$; similarly, $\left(\mathbb{D}_{m}, \widehat{\mathbb{D}}_{m}\right)$ converges weakly to $(\mathbb{D}, \widetilde{\mathbb{D}})$, where $\widetilde{\mathbb{D}}$ is an independent copy of $\mathbb{D}$. Strictly speaking, these two convergence results occur simultaneously. The remainder of the proof would employ arguments very similar to those of Proposition 4.1 , and therefore are omitted.

\section{References}

Anderson, G. (1996). Nonparametric tests of stochastic dominance in income distributions. Econometrica: Journal of the Econometric Society 1183-1193.

Bahraoui, T. and Quessy, J.-F. (2017). Tests of radial symmetry for multivariate copulas based on the copula characteristic function. Electron. J. Stat. 11 2066-2096. MR3652880

Barrett, G. F. and Donald, S. G. (2003). Consistent tests for stochastic dominance. Econometrica 71 71-104. MR1956856 (2003k:62143)

BRAdley, R. C. (2005). Basic properties of strong mixing conditions. a survey and some open questions. Probab. Surv. 2 107-144. Update of, and a supplement to, the 1986 original. MR2178042 (2006g:60054)

BüCHER, A. and Ruppert, M. (2013). Consistent testing for a constant copula under strong mixing based on the tapered block multiplier technique. $J$. Multivariate Anal. 116 208-229. MR3049901

Bücher, A. and Volgushev, S. (2013). Empirical and sequential empirical copula processes under serial dependence. J. Multivariate Anal. 119 61-70. MR3061415

Carrasco, M. and Chen, X. (2002). Mixing and moment properties of various GARCH and stochastic volatility models. Econometric Theory 18 17-39. MR1885348 (2002m:62119)

Cebriàn, A. C., Denuit, M. and Scaillet, O. (2004). Testing for concordance ordering. Astin Bull. 34 151-173. MR2055543

Cook, R. D. and Johnson, M. E. (1981). A family of distributions for modelling nonelliptically symmetric multivariate data. J. Roy. Statist. Soc. Ser. B 43 210-218. MR626768 (83a:62109)

Cook, R. D. and Johnson, M. E. (1986). Generalized Burr-Pareto-logistic distributions with applications to a uranium exploration data set. Technometrics 28 123-131. MR0841485

DAvidson, R. and Duclos, J. Y. (2000). Statistical inference for stochastic dominance and for the measurement of poverty and inequality. Econometrica 68 1435-1464. MR1793365

Denuit, M., Lefevre, C. and Shaked, M. (1998). The $s$-convex orders among real random variables, with applications. Math. Inequal. Appl. 1 585-613. MR1646686 
Denuit, M., Lefèvre, C. and Mesfioui, M. (1999). A class of bivariate stochastic orderings, with applications in actuarial sciences. Insurance Math. Econom. 24 31-50. 1st IME Conference (Amsterdam, 1997). MR1710812

Denuit, M., Lefèvre, C. and Mesfioui, M. (2003). On spline approximation for bivariate functions of increasing convex type. Rev. Anal. Numér. Théor. Approx. 32 145-159.

Denuit, M. M. and Mesfioui, M. (2017). Preserving the Rothschild-Stiglitz type increase in risk with background risk: a characterization. Insurance Math. Econom. 72 1-5. MR3600678

Eubank, R., Schechtman, E. and Yitzhaki, S. (1993). A test for second order stochastic dominance. Comm. Statist. Theory Methods 22 1893-1905. MR1238369

Fernández-Ponce, J. M. and Rodríguez-Griñolo, M. R. (2017). New properties of the orthant convex-type stochastic orders. TEST 26 618-637. MR3683991

Gijbels, I., Omelka, M. and Sznajder, D. (2010). Positive quadrant dependence tests for copulas. Canad. J. Statist. 38 555-581. MR2753002

Goovaerts, M. J., KaAs, R., van HeerwaArden, A. E. and BauWElinckX, T. (1990). Effective actuarial methods. Insurance Series 3. North-Holland Publishing Co., Amsterdam. MR1099819

Guo, X., Li, J., Liu, D. and Wang, J. (2016). Preserving the RothschildStiglitz type of increasing risk with background risk. Insurance Math. Econom. 70 144-149. MR3543040

Hesselager, O. (1996). A unification of some order relations. Insurance Math. Econom. 17 223-224. MR1394770

Joe, H. (2015). Dependence modeling with copulas. Monographs on Statistics and Applied Probability 134. CRC Press, Boca Raton, FL. MR3328438

KosoroK, M. R. (2008). Introduction to empirical processes and semiparametric inference. Springer, New York. MR2724368

Lehmann, E. L. (1986). Testing statistical hypotheses, second ed. Wiley Series in Probability and Mathematical Statistics: Probability and Mathematical Statistics. John Wiley \& Sons Inc., New York. MR0852406 (87j:62001)

Linton, O., Mansoumi, E. and Whang, Y.-J. (2005). Consistent testing for stochastic dominance under general sampling schemes. Rev. Econom. Stud. 72 735-765. MR2148141

Linton, O., Song, K. and Whang, Y.-J. (2010). An improved bootstrap test of stochastic dominance. J. Econometrics 154 186-202. MR2558960

MARShALl, A. W. (1991). Multivariate stochastic orderings and generating cones of functions. In Stochastic orders and decision under risk (Hamburg, 1989). IMS Lecture Notes Monogr. Ser. 19 231-247. Inst. Math. Statist., Hayward, CA. MR1196058

MCFAdDEn, D. (1989). A method of simulated moments for estimation of discrete response models without numerical integration. Econometrica: Journal of the Econometric Society 995-1026. MR1014539

Nelsen, R. B. (2002). Concordance and copulas: a survey. In Distributions with given marginals and statistical modelling 169-177. Kluwer Acad. Publ., 
Dordrecht. MR2058990

Nelsen, R. B. (2006). An introduction to copulas, second ed. Springer Series in Statistics. Springer, New York. MR2197664 (2006i:60004)

RIo, E. (2000). Théorie asymptotique des processus aléatoires faiblement dépendants. Mathématiques et Applications. Springer. MR2117923

RÉmillard, B. and Scaillet, O. (2009). Testing for equality between two copulas. J. Multivariate Anal. 100 377-386. MR2483426

SCAILlet, O. (2005). A Kolmogorov-Smirnov type test for positive quadrant dependence. Canad. J. Statist. 33 415-427. MR2193983

SCARsini, M. (1984). On measures of concordance. Stochastica 8 201-218. MR0796650 (87e:62065)

Schmid, F. and Schmidt, R. (2007). Multivariate extensions of Spearman's rho and related statistics. Statist. Probab. Lett. 77 407-416. MR2339046

SkLAR, A. (1959). Fonctions de répartition à $n$ dimensions et leurs marges. Publ. Inst. Statist. Univ. Paris 8 229-231. MR0125600

TCHen, A. H. (1980). Inequalities for distributions with given marginals. Ann. Probab. 8 814-827. MR0577318 (82f:60052)

Whitt, W. (1976). Bivariate distributions with given marginals. Ann. Statist. 4 1280-1289. MR0426099

Yanagimoto, T. and Окамото, M. (1969). Partial orderings of permutations and monotonicity of a rank correlation statistic. Ann. Inst. Statist. Math. 21 489-506. MR0258209 (41 \#\#2856) 